\title{
The risk of tuberculosis in transplant candidates and recipients: a TBNET consensus statement
}

\author{
Dragos Bumbacea, Sandra M. Arend, Fusun Eyuboglu, Jay A. Fishman, Delia Goletti, \\ Michael G. Ison, Christine E. Jones, Beate Kampmann, Camille N. Kotton, \\ Christoph Lange, Per Ljungman, Heather Milburn, Michele I. Morris, Elmi Muller, \\ Patricia Muñoz, Anoma Nellore, Hans L. Rieder, Urban Sester, \\ Nicole Theodoropoulos, Dirk Wagner and Martina Sester
}

AFFILIATIONS

For a full list of the authors affiliations, see the Acknowledgements section.

CORRESPONDENCE

M. Sester

Dept of Transplant and Infection Immunology

Saarland University

Kirrberger Strasse

D-66421 Homburg

Germany

E-mail: martina.sester@uks.eu

Received:

Jan 032012

Accepted after revision:

Feb 282012

First published online:

April 102012
KEYWORDS: Guideline, management, Mycobacterium tuberculosis, transplantation, tuberculosis

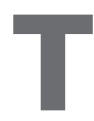
uberculosis (TB) is caused by the pathogenic species of the Mycobacterium tuberculosis complex. Only a minority of individuals who develop an adaptive immune response following infection with $M$. tuberculosis will ever develop TB, with the actual risk depending on the extent to which the host immune system provides a successful or inadequate response $[1,2]$. Therefore, individuals with impaired immune response, such as solid organ transplant (SOT) and hematopoietic stem cell transplant (HSCT) recipients, are more prone to develop TB than immunocompetent persons.

TB in transplant recipients is more frequent compared to the general population (estimates from the last decades state 20-74 times as frequent in SOT [3, 4] and twice as frequent in HSCT [5]), and more often fatal (up to $31 \%$ in SOT [6] and up to $50 \%$ in
HSCT recipients [7]), thus adding effectiveness to interventions for its prevention, even in the face of difficulties, with treatment related to adverse drug events and drug-drug interactions. Active TB in transplant recipients can result from latent infection with $M$. tuberculosis (LTBI) in the transplant candidate or in the donor tissue, or from de novo post-transplant infection. These various scenarios prompt for targeted pre-transplant screening of both recipient and, if possible, donors to allow focused management of recipients selected for preventive intervention in the pre- and/or posttransplant period. The term "preventive chemotherapy" is used to denote treatment of LTBI among individuals identified as having a positive immune response to $M$. tuberculosis antigens. "Chemoprophylaxis" is used here to denote primary chemoprophylaxis to prevent TB among individuals with
European Respiratory Journal Print ISSN 0903-1936 Online ISSN 1399-3003 
risk factors (i.e. exposure to $M$. tuberculosis, living in or originating from highly endemic regions, etc.) but without a positive immune response to $M$. tuberculosis antigens (either negative test or not tested).

Diagnosis and treatment of active TB in transplant recipients is challenging due to: 1 ) diverse pulmonary and non-pulmonary conditions mimicking $\mathrm{TB}$; 2) more frequent adverse events from first-line anti-TB drugs; and 3) significant interactions with immunosuppressive drugs.

Direct evidence to support management of transplant recipients for prevention and treatment of TB is often lacking and decisions are largely based on expert opinion and extrapolation from immunocompetent and other immunocompromised populations. This review was elaborated by a TBNET consensus group and summarises current evidence and clinical experience of the authors on TB prevention and treatment in SOT and HSCT recipients. It provides evidence-graded recommendations (evidence categories A-D) (table 1) for screening and diagnosis of LTBI, preventive chemotherapy/chemoprophylaxis and treatment of active TB before and after transplantation.

\section{NATURAL HISTORY OF M. TUBERCULOSIS INFECTION IN TRANSPLANT CANDIDATES AND RECIPIENTS The natural history of $\mathrm{M}$. tuberculosis infection}

Infection with $M$. tuberculosis is almost exclusively acquired by inhalation of bacilli-containing droplet nuclei. Inhaled bacteria are engulfed by polymorphonuclear granulocytes and alveolar macrophages but have mechanisms to survive. In this early phase, there is potentially lymphogenic and haematogenic spread of $M$. tuberculosis to the lungs, as well as to other organs and tissues, until the specific immune response arrests further multiplication of bacilli, contains the infection and, ultimately, eliminates bacilli in the majority of cases. Antigen-specific and -nonspecific signals from $M$. tuberculosis-infected macrophages stimulate T-cell immunity. The mechanisms of immune control of $M$. tuberculosis require a complicated collaboration between different cell types that is only partly understood [8]. The role of a T-helper (Th)1 response, especially of CD4 and CD8 interferon (IFN)- $\gamma$ producing T-cells, is considered essential. Polyfunctional memory CD4 T-cells that simultaneously produce IFN- $\gamma$, tumour necrosis factor (TNF)- $\alpha$ and interleukin (IL)-2 appear to be mediators of long lasting protection against TB reactivation [9-13]

Probably $\sim 1 \%$ of putatively infected individuals, other than infants and small children, will have directly progressive active TB within the first year following primary infection. In most otherwise healthy individuals, specific cellular immune responses are sufficient to prevent the progress to TB. A clinically pragmatic definition of LTBI is the presence of a specific immune response detected by the tuberculin skin test (TST) or an IFN- $\gamma$ release assay (IGRA), in the absence of TB [14]. Changes in gene expression of granuloma encased bacilli are related to a state of low metabolic activity in which $M$. tuberculosis can survive for prolonged periods of time $[15,16]$. The cumulative lifetime risk of reactivation TB is estimated at $\sim 5-10 \%[17,18]$, but varies depending on factors, notably age at infection and remaining lifetime and immune status. The risk is $\sim 5 \%$ in the first $5-7$ yrs after infection decreasing to $\sim 0.1 \%$ per year thereafter. The risk may persist for a lifetime as exemplified by a documented interval of $>50$ yrs $[19,20]$. While there are recognised risk factors of reactivation, in most persons with reactivation TB there is no known risk factor. Age is relevant, as TB occurs more frequently among adolescents and young adults. The risk for disease might also be increased among very old persons, but apart from increasing age seemingly playing a role, little is known about the modifiers of age- and sex-specific risk. Except for rare inherited immunodeficiencies [21], the role of genetic factors as determinants of the risk of TB is probably limited compared to the level of exposure per se [22].

\section{Pathogenesis of TB in the transplant setting}

In addition to other recognised factors, the risk of active TB in a transplant recipient is related to infection and re-infection with $M$. tuberculosis and the net state of immunosuppression. When the

\section{TABLE 1 Description of levels of evidence}

\begin{tabular}{|c|c|c|}
\hline Evidence category & Sources of evidence & Definition \\
\hline A & $\mathrm{RCT}$; rich body of data & $\begin{array}{l}\text { Evidence is from end-points of well-designed RCTs that provide a consistent pattern } \\
\text { of findings in the population for which the recommendation is made } \\
\text { Category A requires substantial numbers of studies involving substantial numbers } \\
\text { of participants }\end{array}$ \\
\hline B & RCT; limited body of data & $\begin{array}{l}\text { Evidence is from end-points of intervention studies that include only a limited } \\
\text { number of patients, post-hoc or subgroup analysis of RCTs, or meta-analysis of RCTs } \\
\text { In general, category B pertains when few randomised trials exist, they are small in size, } \\
\text { they were undertaken in a population that differs from the target population of the } \\
\text { recommendation, or the results are somewhat inconsistent }\end{array}$ \\
\hline C & $\begin{array}{l}\text { Non-randomised trials; } \\
\text { observational studies }\end{array}$ & $\begin{array}{l}\text { Evidence is from outcomes of uncontrolled or non-randomised trials or from } \\
\text { observational studies }\end{array}$ \\
\hline D & Panel consensus judgement & $\begin{array}{l}\text { This category is used only in cases where the provision of some guidance was deemed } \\
\text { valuable but the clinical literature addressing the subject was insufficient to justify } \\
\text { placement in one of the other categories } \\
\text { The Panel consensus is based on clinical experience or knowledge that does not } \\
\text { meet the criteria listed above }\end{array}$ \\
\hline
\end{tabular}




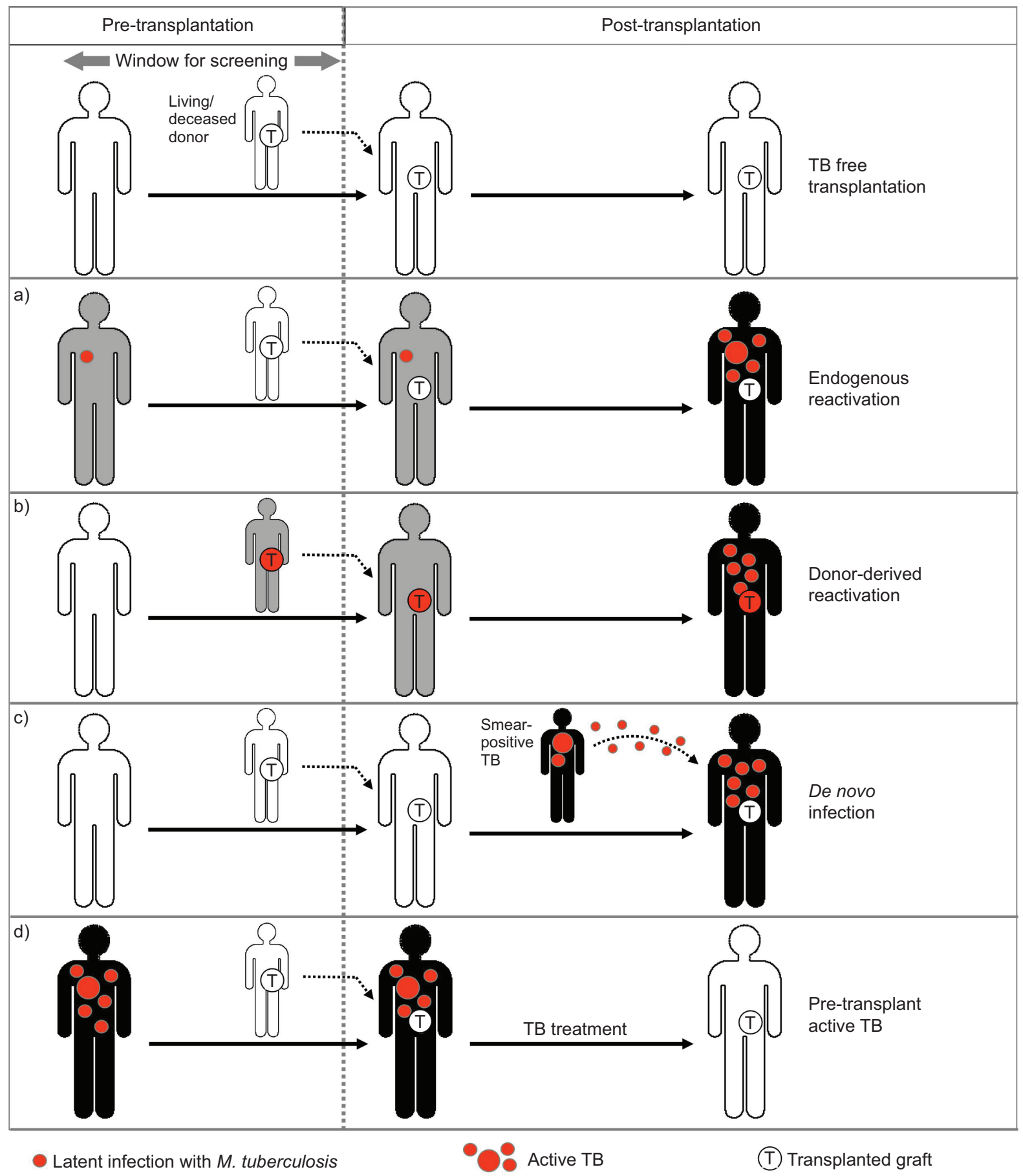

FIGURE 1. The four different scenarios for infection with Mycobacterium tuberculosis in the transplant setting. a) Endogenous reactivation due to latent infection with M. tuberculosis (LTBI) in the candidate recipient. b) Donor-derived reactivation due to LTBI in a living or deceased donor. c) De novo exposure and infection posttransplantation. d) When a patient with active TB urgently requires a transplant (i.e. urgent liver transplantation). White, grey and black figures represent uninfected individuals, individuals with LTBI, and individuals with active TB, respectively.

cellular immune system is impaired due to disease or treatment, the risk is increased for all three contributory components of TB morbidity, namely primary $\mathrm{TB}$ resulting from progression of an infection acquired in the past $5 \mathrm{yrs}$, endogenous reactivation disease of a distant infection acquired $>5$ yrs previously, and exogenous acquisition of a recent or distant infection [23]. In general, active TB is associated with four different scenarios in transplant recipients (fig. 1).
Scenario A: latent infection in the candidate

When a transplant candidate is latently infected with $M$. tuberculosis, this offers a window of opportunity for detection and preventative treatment (fig. 1a). Similar to immunocompetent individuals, where the risk of TB decreases with increasing time after infection, the risk of post-transplantation reactivation is probably inversely related to the interval between infection and transplantation, but this is hard to prove as the interval is rarely known. 


\section{Scenario B: infection in the donor graft}

The donor organ or tissue may be infected with $M$. tuberculosis, which can be the source of donor-derived transmission (fig. 1b). Transmission via lung transplantation is most likely and best documented [24], since involvement and pathogen load may be higher; although LTBI may reactivate from other infected grafts $[25,26]$, risk estimates are less clear. Treatment of donors with recognised LTBI or risk factors for LTBI may reduce the risk of reactivation.

In scenarios A and B, the risk of reactivation is probably highest early post-transplant given enhanced immunosuppression; later onset may also occur with intensification of immunosuppression, such as during the treatment of rejection.

\section{Scenario C: de novo infection post transplant}

Transplant recipients who are exposed to and become infected with $M$. tuberculosis post transplantation have a very high risk of immediate progression. Unfortunately, exposure that may lead to infection is not always recognised and neither is a recently acquired infection, and even if it is recognised the available immunodiagnostic tests for screening are less reliable in immunosuppressed patients.

\section{Scenario D: candidate with active TB requiring urgent transplantation}

Rarely, a patient with active TB is in urgent need of transplantation, such as the result of drug-induced hepatotoxicity resulting in serious liver injury [27]. The special considerations regarding the treatment of active $\mathrm{TB}$ after transplantation are discussed further later.

As most cases of post-transplantation TB originate from a focus of LTBI, a better understanding of latency is needed. One model supported by animal data is that latent infection is not a stationary phase, but rather a cyclic change between replication and immune containment, with a progressive increase in the length of the interval between successive episodes [28]. If correct, this model would explain the decreasing risk of reactivation over time and also why prolonged treatment is needed to eradicate just a small number of bacilli, isoniazid being an inhibitor of cell wall synthesis and therefore active only during rare episodes of heightened metabolic activity. Although it is possible that a substantial proportion of individuals may eliminate tubercle bacilli acquired in the past [29], all currently available tests measure a long lasting immunologic response that is only partially dependent on the continued presence of live bacilli (exemplified by a persisting positive TST reaction remaining positive years after bacilli Calmette-Guérin (BCG) vaccination). Therefore, it is not known how to specifically identify individuals where true LTBI has not yet died out. Such information would allow more targeted treatment of candidate transplant donors or recipients.

\section{Summary}

1. Infection with $M$. tuberculosis in the transplant setting is relevant in four different scenarios, which are: a) LTBI in the candidate recipient, b) LTBI in a living or deceased donor, c) de novo exposure post transplantation, and d) when a patient with active TB urgently requires a transplant.

2. The prevention of active TB is not always possible as it depends on reliable detection and treatment of true LTBI in recipients or donors and awareness of post-transplantation exposure.
3. If the a priori risk of M. tuberculosis infection is high, therapeutic decisions may have to be made without evidence.

\section{RISK OF TB IN SOT RECIPIENTS}

TB remains a rare but clinically significant complication of SOT recipients [4]. The rates and risk of TB in transplant recipients are highly dependent upon the key features, such as the frequency of TB in the recipient and donor population, the organ transplanted (highest in lung transplant recipients), the type and intensity of recipient screening for $\mathrm{TB}$, and the use of prior or current anti-TB drug intake, preventively or curatively $[4,30,31]$.

The rate of $\mathrm{TB}$ in transplant recipients is highly linked to endemicity of $M$. tuberculosis infection. In the USA and much of Western Europe, the general incidence of TB is currently low and ranges between 0 and 24 cases per 100,000 population, whereas incidences may be as high as or higher than $1 \%$ per annum in South Africa [32]. The overall frequency of TB in transplant populations has been estimated to be roughly between 20 and 74 times that of the general population [3, 4]. In areas with low endemicity of $\mathrm{TB}$, the prevalence among SOT recipients is $0.5-6.4 \%$, while it has been reported to be as high as $15.2 \%$ in highly endemic areas [3,31]. As such, differential preventative strategies are needed for donors or recipients from regions with varying endemicity.

Apart from general endemicity, the rate of TB is heavily dependent upon the organ transplanted and the level of immunosuppression involved [3]. There are no data to clearly associate an effect of a specific drug with risk for TB. This is mainly due to the fact that a minority of patients take single agents for immunosuppression. Although TB may affect any transplanted patient, recipients of lung transplants have the highest rate of TB relative to other transplanted organs (relative risk 5.6) [33]. This is not surprising since the lung is the primary portal of entry for $M$. tuberculosis. Other factors that may affect the incidence of TB include the use of T-cell depleting antibodies, enhanced immunosuppression in the setting of rejection, chronic renal insufficiency or haemodialysis for kidney transplant recipients, diabetes mellitus, hepatitis $C$ virus infection for kidney transplant recipients, chronic liver disease, or increased recipient age [3, 30,33].

The majority of TB cases occur during the first 6 months posttransplant, except in renal transplant patients, where onset is typically later [3]. A history of a positive TST or radiographic evidence of past TB is associated with earlier onset of disease [3]. A greater proportion of patients who receive lymphocytedepleting antibodies have an earlier onset of TB than those who receive other types of induction therapies [3]. Although most transplant patients present with pulmonary TB (51\%), 16\% have extrapulmonary disease of specific organs and 33\% have disseminated TB [3]. Hallmark symptoms of TB, i.e. fever and constitutional symptoms, such as night sweats and weight loss, occur frequently but not universally in transplant patients. Fever, for example, was seen in $64 \%$ of transplant recipients with localised disease and in $91 \%$ with disseminated disease [3]. Atypical presentations may frequently occur, particularly in patients with disseminated TB; such presentations may be more common in donor-derived TB transmission [25]. In patients with pulmonary disease, a wide range of radiographic manifestations, including focal infiltrate, miliary pattern, nodules, pleural effusions, diffuse interstitial infiltrates and cavitary disease have 
been described $[3,6,30]$. As a result, of the myriad of presentations, up to a third of patients may not have TB suspected initially and $3-5 \%$ may only be diagnosed after the death of the recipient $[3,6]$.

The risk of adverse outcomes following TB among SOT recipients is increased compared to non-immunocompromised patients. Mortality rates of $19-40 \%$ have been described which represents a 10-fold increase compared to the overall mortality of TB $[3,6,30,33,34]$. Most fatality $(57-83 \%)$ in these patients is directly attributable to TB [3]. In addition, complex interactions between the agents used to treat TB and the agents typically used to prevent rejection may result in allograft loss in up to one third of cases $[6,35]$. Additional features contributing to an increased morbidity and mortality include the difficulty in early diagnosis resulting in a delay in initiation of therapy, and increased incidence of disseminated disease at the time of diagnosis.

\section{Summary}

1. The prevalence of post-transplant TB is lower in lowendemicity areas $(0.5-6.4 \%)$ than in areas of higher endemicity for TB (up to $15.2 \%$ ).

2. The rate of post-transplant TB is heavily dependent upon the organ transplanted (highest for lung transplant recipients).

3. Other risk factors for post-transplant ТВ include use of lymphocyte depleting antibodies, enhanced immunosuppression, chronic renal insufficiency or haemodialysis, diabetes mellitus, hepatitis $C$ virus infection in kidney transplant recipients, chronic liver disease, increased recipient age, and positive TST or IGRA results pre-transplant.

4. Although pulmonary disease may occur, other clinical presentations, including fever of unknown origin or septicaemia, may occur post-transplant.

5. Generally, transplant recipients have a higher rate of morbidity, mortality and graft loss if TB complicates the posttransplant course.

\section{DIAGNOSIS OF LATENT INFECTION WITH M. TUBERCULOSIS IN TRANSPLANT CANDIDATES, DONORS AND RECIPIENTS}

Detection of a cellular immune response against $M$. tuberculosisspecific antigens is the commonly accepted indirect measure of possible infection. This response is not per se indicative of the presence of viable bacilli and thus risk for future TB $[14,36]$. The cellular immune response to $M$. tuberculosis may be analysed by the use of two test principles, the in vivo TST and the ex vivo IGRA (fig. 2). The TST elicits a delayed-type hypersensitivity (DTH) response after local intradermal application of purified protein derivative (PPD) [37]. The diameter of induration is quantified 48$72 \mathrm{~h}$ after antigen injection [38] with specific cut-off values for positive test results that are defined to provide the putatively optimised value to balance deficiencies in sensitivity and specificity, respectively. Preference is given to sensitivity at the cost of specificity if the expected risk is particularly high, such as in the case of immunodeficiency of an individual [39], but whether this trade-off is as efficient as postulated has been called into question, at least in HIV-associated immunodeficiency [40]. More recently, bloodbased IGRAs have been developed and evaluated for the risk estimation of TB in clinical routine [41]. As with TST, IGRAs rely on specific stimulation of effector T-cells that are activated to produce cytokines within hours after stimulation, and IFN- $\gamma$ is most commonly used as readout for specific activation of T-cells [42].

IFN- $\gamma$ production may be analysed by a variety of experimental assays (fig. 2). The amount of IFN- $\gamma$ may either be analysed in the supernatants of stimulated whole blood cells using an ELISA [43] or IFN- $\gamma$ producing T-cells may be quantified from isolated peripheral blood mononuclear cells (PBMC) using the enzymelinked immunospot assay (ELISPOT) [44, 45]. Commercial tests are available for both formats (QuantiFERON TB ${ }_{\mathbb{R}}$ Gold in-tube; Cellestis, Victoria, Australia, and T-SPOT.TB ${ }_{\mathbb{B}}$; Oxford Immunotec, Oxford, UK). More recently, flow cytometry has been used to quantify specifically activated T-cells based on the intracellular accumulation of IFN- $\gamma$ or other cytokines. Although this technique has so far largely been applied in experimental settings, its potential to perform multi-parameter analyses may hold promise as an immune-based technique to distinguish LTBI from active disease $[9,11-13]$.

When compared to TST, IGRAs have some operational advantages that are particularly relevant in immunocompromised patients. Unlike the TST, antigen-specific stimulation in vitro is carried out along with negative and positive controls. As the positive control allows for assessment of general T-cell responsiveness, in vitro tests may be able to discriminate true negative responses from anergy and/or overt immunosuppression. Further advantages of IGRAs may result from an increase in specificity in the face of increased, or at least similar, sensitivity [46-48]. An increase in specificity is due to the use of antigens derived from $M$. tuberculosis that are absent in all strains of $M$. bovis BCG and most environmental mycobacteria, including species from the $M$. avium complex [42, 49, 50]. These antigens, the early secretory antigenic target (ESAT)-6, culture filtrate protein (CFP)-10 (both region of difference (RD)1-derived) or TB7.7 (RD11-derived), elicit a T-cell response that is a more specific marker for $M$. tuberculosis infection than a TST response towards PPD, especially in BCG-vaccinated individuals $[48,51]$. In vitro assays may also hold promise as a more sensitive alternative to TST in immunocompromised patients, where skin testing may frequently be falsely negative and thus of low negative predictive value for disease progression [52-54]. In the setting of transplantation, this is largely attributed to the combined action of various immunosuppressive drugs that interfere with T-cell function that may affect both TST and IGRAs (fig. 2). Corticosteroids inhibit the function of both T-cells and antigenpresenting cells, anti-metabolite drugs interfere with proliferative capacities of T-cells, calcineurin inhibitors directly inhibit induction of T-cell cytokines, and finally depleting antibodies used as induction or rejection therapy result in the elimination of T-cells [55]. Likewise, the uraemia-associated immunodeficiency syndrome in transplant candidates with end-stage renal failure has been characterised by a defect in co-stimulatory activity to antigen-specific T-cells that may directly contribute to a decrease in T-cell function $[56,57]$. Studies on the use of IGRAs in transplant recipients are rare and frequently inconclusive. In theory, immunosuppressive drugs should equally interfere with T-cell reactivity in skin testing and in IGRAs; yet evidence from experimental and clinical studies indicate that in vitro assays are less affected in patients with moderate immunosuppression [58]. If patients receive higher levels of immunosuppressive drugs and/ or are severely T-cell depleted, however, caution is warranted 
Skin test

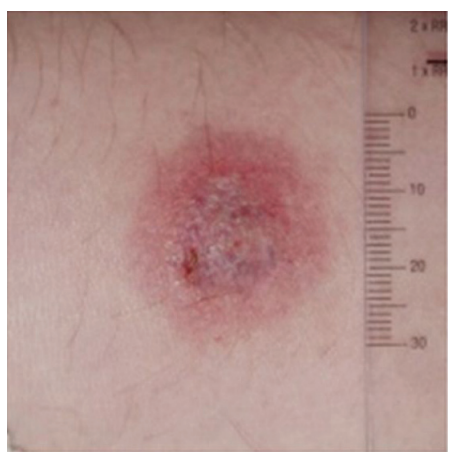

ELISA

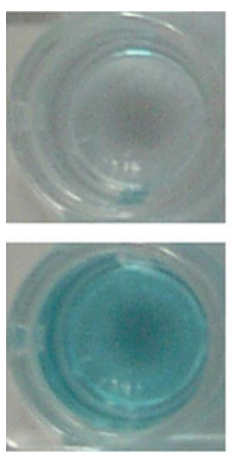

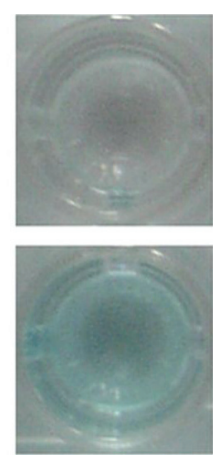

ELISPOT assay

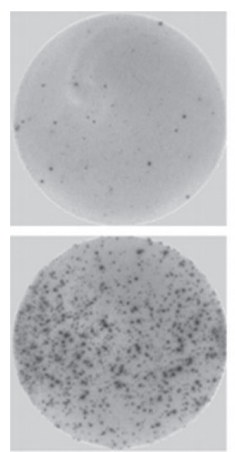

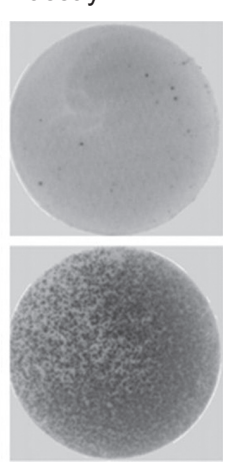

Flow cytometry

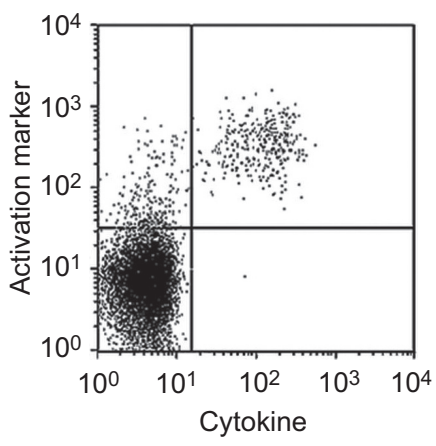

Cytokine induction

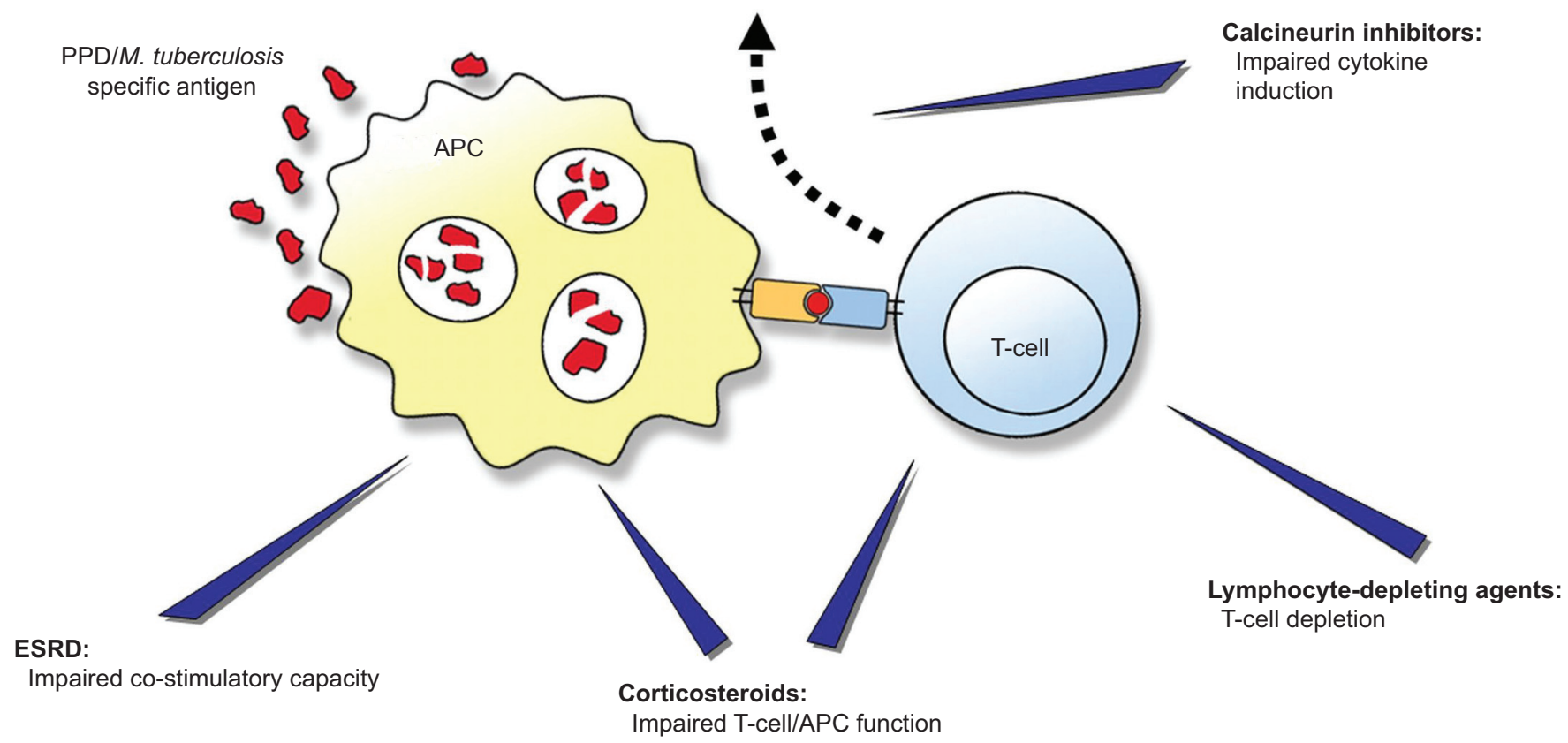

FIGURE 2. Immune-based T-cell assays for the diagnosis of a latent infection with Mycobacterium tuberculosis. All tests rely on stimulation with either purified protein derivative (PPD) or M. tuberculosis specific antigens that elicit a cytokine induction in specific T-cells. Cytokines may be detected in vivo by skin testing or in vitro by interferon- $\gamma$ release assays: ELISPOT assay, ELISA or flow cytometry. End-stage renal disease (ESRD) as well as currently used immunosuppressive drugs or lymphocyte-depleting agents applied in solid organ transplant or hematopoietic stem cell transplant have various mechanisms to interfere with T-cell reactivity. APC: antigen presenting cell.

with the use of IGRAs as they may yield falsely negative diagnoses or indeterminate results [58,59]. As a consequence, the sensitivity of IGRAs varies between studies and is dependent on the overall level and mode of immunosuppression. Positive and negative predictive values of immunodiagnostic tests in the transplant population are influenced by the locality-specific prevalence of infection with $M$. tuberculosis and they are likely to differ from respective estimates in other risk groups for TB or healthy contacts, a key determinant being age differences and thus accumulated risk of being infected with M. tuberculosis.

In the setting of transplantation, screening for LTBI in both the recipient and the donor may allow assessment of the risk of developing TB after transplant; positive screening results in individuals from high-risk groups for the development of TB should prompt for a decision for preventive chemotherapy $[60,61]$. While screening of living donors is achievable, testing of deceased donors is challenging, as TST is not feasible and the performance of in vitro assays have not yet been assessed. When screening recipients, the decrease in test sensitivity with increasing immunosuppression has important practical consequences, as screening should be carried out before administration of immunosuppressive drugs to ensure sensitivity and to allow sufficient time to initiate chemoprophylaxis. Studies in transplant candidates prior to liver $[62,63]$ or renal transplantation indicate that IGRAs may be applied, although agreement between TST and IGRAs in renal transplant candidates is only fair to moderate [64-68].

At present, the advantage of either test for risk assessment posttransplant is not known, as the positive predictive value of a positive IGRA for the development of TB has not been sufficiently studied. The limited number of studies so far indicates that its value may be higher in low-prevalence countries [69-72] as compared to highly endemic regions [46, 73]. At present, recommendations that favour the use of IGRAs over TST in 
immunocompromised patients are largely based on potential superiority in identifying individuals with LTBI. Notably, a recent prospective study in South Korea showed that four out of 272 TST-negative renal transplant candidates developed TB posttransplant. All four individuals had a positive ELISPOT assay, whereas no TB developed in patients with negative or indeterminate results [74]. In addition, a recent retrospective analysis among 461 immunocompromised patients showed that one out of 38 QuantiFERON-TB gold (QFT) positive and no QFT-negative patients developed active TB [75]. While this clearly indicates an improved identification of patients at risk for $\mathrm{TB}$, more studies in both low- and high-prevalence regions are needed to comparatively assess the positive predictive values of different immunodiagnostic tests in the setting of transplantation.

\section{Summary}

1. TST measures a cell-mediated immune response to tuberculin PPD and has poor specificity in patients with BCG vaccination given in the not too distant past.

2. IGRAs are blood-based ex vivo tests that rely on the rapid induction of IFN- $\gamma$ in response to antigens that are more specific for the detection of a latent M. tuberculosis infection than PPD.

3. IGRA testing in living donors, transplant candidates and transplant recipients is feasible, although sensitivity may decrease with increasing immunosuppression; knowledge of IGRA performance in deceased donors is lacking.

4. Further longitudinal studies are needed to estimate the risk for progression to post-transplant TB after IGRA- and TSTbased screening.

\section{PREVENTION OF TB IN TRANSPLANT RECIPIENTS}

Effective pre-transplant screening for LTBI may prevent significant morbidity and mortality post-transplant by identifying individuals at risk for reactivation disease. Apart from TST- or IGRA-positive individuals after targeted screening, treatment of SOT recipients may also be indicated due to a high-risk pretransplant exposure history (even with negative TST or IGRA), residence in an endemic TB region during the early posttransplant period, specific M. tuberculosis exposure post-transplant, or with a donor history of untreated or incompletely treated LTBI or TB [31, 76]. In endemic areas, some centres administer isoniazid for a period of time after transplant [31, 77]. Recommendations for management of various clinical situations, including involvement of donors with $\mathrm{TB}$, are described later. Although treatment of LTBI in transplant candidates is complicated by the presence of organ failure, with careful monitoring it can generally be safely initiated before or early after transplantation.

The risk of TB is highest in the first year post-transplant, during the time of maximal immunosuppression, with a median onset at 9 months [3]. Transplant recipients are at risk of other opportunistic infections at this time, and the diagnosis may therefore be overlooked, especially in areas of low-TB endemicity, resulting in delayed treatment and poor outcomes [78]. Risk-adapted pretransplant screening is therefore essential, not only to provide the opportunity for preventive chemotherapy in patients with LTBI, but also to raise the index of suspicion of active disease in those patients at high risk. In cases where the pre-transplant screening may be falsely negative, as with anergy to TST or an indeterminate IGRA, specialised imaging may be employed in selected patients. Thoracic computed tomography (CT) scanning may be superior to chest radiography in assessing the risk of post-transplant TB [79]. Genitourinary studies may also be helpful in higher risk recipients. Transplant candidates should not begin therapy for LTBI until they have been clinically evaluated for active TB, both pulmonary and extra-pulmonary [30]. It is strongly recommended

TABLE 2 Monitoring of latent tuberculosis infection (LTBI) treatment in solid organ transplant candidates/recipients [39, 84]

Timing of intervention

Prior to onset of LTBI treatment

Monthly during therapy ${ }^{\#}$

Physical examination

With signs or symptoms of hepatotoxicity

Discontinue medication

Discontinue medication
Physical examination

Rule out active TB through careful history, physical examination and testing

Emergent evaluation with physical examination

Signs or symptoms of hepatotoxicity (severe anorexia, rash, nausea, vomiting, jaundice, dark urine, right upper quadrant pain) or new symptoms of peripheral neuropathy: $>3$ days of unexplained fever and weakness

No signs or symptoms

\section{Laboratory testing}

Rule out active TB by thoracic imaging using CXR; consider thorax $\mathrm{CT}$ if high suspicion of $\mathrm{LTBI}$ Evaluate sputum AFB smear and culture and PCR if appropriate Evaluate urine AFB and genitourinary imaging if high suspicion of genitourinary TB infection

AST, ALT and bilirubin

AST, ALT and bilirubin

Drug levels of medications with high risk for drug interaction AST, ALT and bilirubin

Drug levels of medications with high risk for drug interaction Strongly consider withholding medication while awaiting results AST or ALT more than three times the upper limit of normal with symptoms

AST or ALT more than five times the upper limit of normal without symptoms

TB: tuberculosis; CXR: chest radiography; CT: computed tomography; AFB: acid fast bacilli; AST: aspartate transaminase; ALT: alanine transaminase. ${ }^{\#:}$ may begin follow-up 2 weeks after initiation of therapy in high-risk liver patients. 
that a physician with expertise in transplant infectious diseases be involved in this evaluation and subsequent therapy.

Treatment of LTBI in transplant candidates should be offered according to national guidelines, as these generally reflect regional drug availability and resistance patterns [39]. Potential regimens include, alone or in combination, isoniazid, rifampicin and a fluoroquinolone. The combination of rifampicin and pyrazinamide has been associated with significant hepatotoxicity and should be used as an exception and with close monitoring of hepatic function [80-83]. Treatment should be initiated as early as possible pre-transplant, depending on the patient's medical condition and hepatic function. Careful monitoring for multiple potentially significant drug interactions is required, as well as close follow-up for adverse drug events, which may be significant in transplant candidates with end organ failure (table 2).

Timing of preventive chemotherapy/chemoprophylaxis in SOT recipients has not been well studied. Some clinicians prefer to administer it in the pre-transplant period as it lowers the risk of drug interactions. However, others prefer to use it after transplant, once the immunosuppression has started and the risk of reactivation TB is higher. This can be problematic as there is more potential for drug interactions, especially with the rifamycins (table 3). Transplant recipients are generally monitored closely in the first year after transplant, which may improve safety through close therapeutic monitoring.

The choice of agent(s) for preventive chemotherapy/chemoprophylaxis may depend on local rates of anti-TB drug resistance of M. tuberculosis isolates, organ disease, drug or drug combination toxicity, drug interactions, likelihood of adherence and other factors. Again, it is recommended that transplant programmes follow the recommendations of local or governmental programmes. Commonly used regimens include isoniazid for 9 months (often given with pyridoxine) or rifampicin for 4 months. When toxicity, resistance or drug interactions preclude safe use of those drugs, preventive chemotherapy with a fluoroquinolone could be considered appropriate by some authorities. Clinicians caring for transplant recipients should be aware that administration of isoniazid or rifampicin significantly reduces the risk of development of $\mathrm{TB}$ depending on the duration the drugs are administered alone or in combination, but that preventive chemotherapy may not eliminate all viable M. tuberculosis bacilli. Reactivation of TB may have an atypical presentation in the immunosuppressed host, and may occur both in pulmonary and extrapulmonary sites.

In patients awaiting heart transplantation, concomitant isoniazid and warfarin therapy may be initiated in cardiomyopathy patients, although it requires significant coordination with the cardiology team to ensure that appropriate monitoring of anticoagulation occurs. Alternative regimens such as rifamycins may decrease international normalised ratio (INR), increasing the risk of clotting. Fluoroquinolones may prolong the QT interval in patients at risk for arrhythmias.

The presence of hepatitis or end-stage liver disease may significantly complicate the use of either isoniazid or rifampicin pre-transplant. Careful and frequent monitoring for signs and symptoms of drug-induced liver injury is strongly recommended in all patients being treated for LTBI, in conjunction with monthly liver enzyme testing throughout treatment [85]. More frequent monitoring may be indicated in high-risk patients, particularly older patients and those with potential for significant drug-drug interactions. There are some data to suggest that hepatotoxicity may be less frequent with a shorter duration of the rifampicin regimen, a potentially important consideration for patients awaiting liver transplantation [86]. The optimal length of LTBI treatment has not been studied in this population. In general, it is recommended that transplant centres follow the recommendations of local or governmental programmes. In some situations, treatment may be given for longer periods of time, especially in more intensely immunosuppressed transplant recipients, or where the risk of further acquisition of $\mathrm{TB}$ is higher (i.e. in endemic regions). Prolonging therapy of LTBI may be desired, but increases the risk of potential complications. TB treatment decisions should be individualised with the assistance of transplant infectious disease expertise.

The optimal timing of LTBI treatment in transplant candidates cannot always be achieved, due to the complex drug interactions and organ dysfunction complicating the management of many patients with organ failure. It is not known what duration of treatment interruption is safe without incurring a substantially increased risk for active TB. Physicians must balance the risk of TB post-transplantation with the risk of deferring an organ offer in a seriously ill patient. It is usually possible to complete the course of LTBI therapy in most renal transplant recipients. Some practitioners prefer to defer treatment of liver transplant candidates and begin post-transplant, although others report successful treatment of patients with liver disease prior to surgery $[87,88]$. If treatment is started pre-transplant and not completed by the time of transplant, re-initiation is recommended as soon as the patient can tolerate and absorb medications after surgery, providing that organ function is stable and with close attention to potential drug interactions. Prolonged treatment interruptions may require an extension of the total duration of LTBI posttransplant. If LTBI therapy is interrupted for $>2$ months, the organ recipient should be rechecked for evidence of active TB before single drug therapy is resumed [39, 89].

\section{Summary}

1. Pre-transplant screening for LTBI may be performed with TST and/or IGRA, although some transplant candidates with LTBI may have false-negative results despite a high-risk pretransplant exposure history. Consider thoracic imaging and/or LTBI therapy for such candidates.

2. Evaluate carefully to exclude active TB before initiating single drug therapy for LTBI.

3. Consider treatment of LTBI pre-transplant, with careful monitoring for drug toxicity or interactions, particularly in patients with organ failure.

4. Chemoprophylaxis/preventive therapy for LTBI need not be completed before transplant, and should be re-initiated as soon as the patient is stable after transplant; treatment interruptions may require a reassessment for development of active TB and an extended duration of LTBI therapy.

\section{TREATMENT OF ACTIVE TB IN TRANSPLANT RECIPIENTS}

Prompt anti-TB therapy should be initiated in patients with proven or probable $\mathrm{TB}$, based on epidemiology, as well as on clinical, 


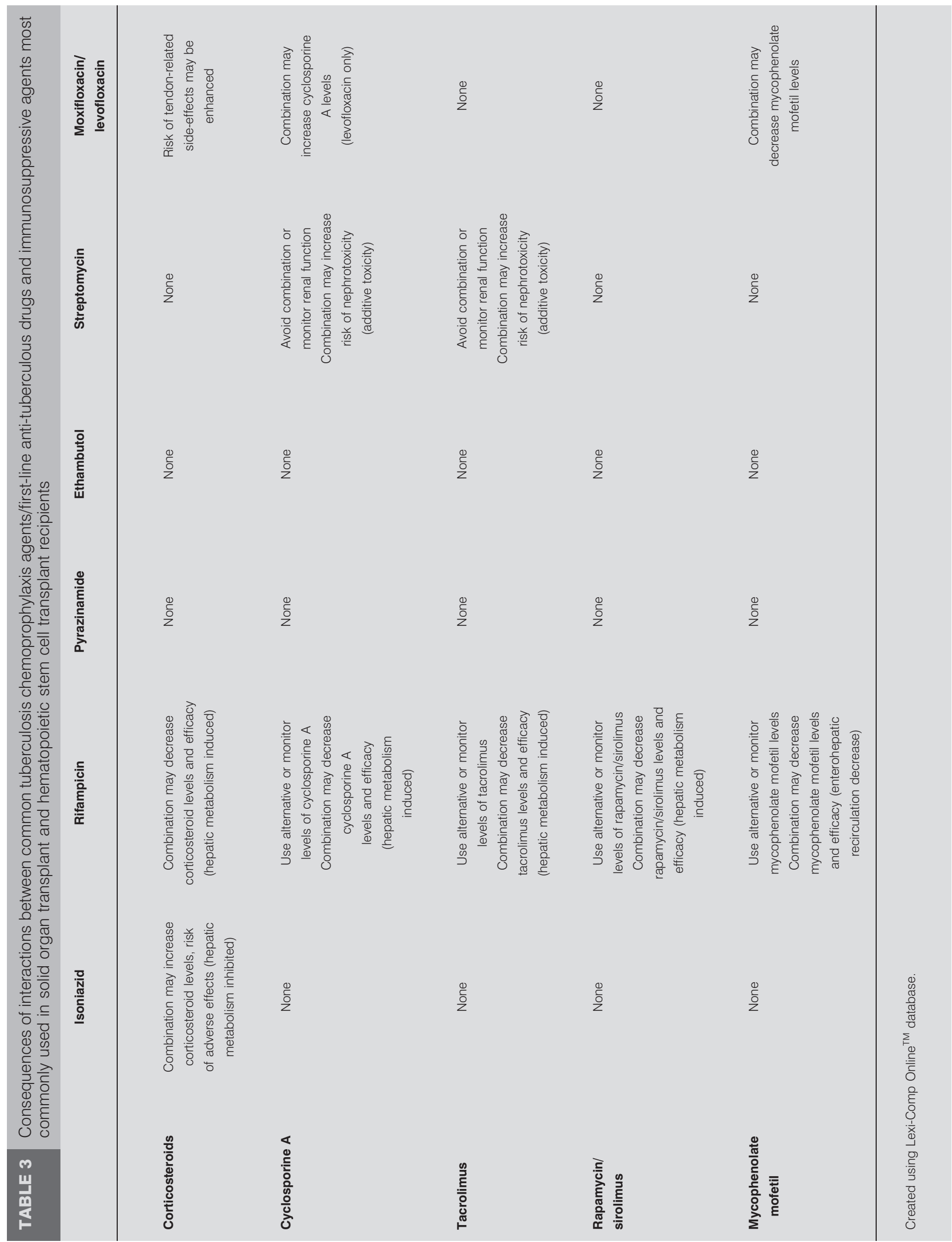


radiographic, microbiological, molecular biological and histopathological tests. Consequently, patients with clinical symptoms of weight loss, fever and sweats should receive immediate anti-TB treatment unless contraindicated. TB treatment in transplant recipients differs from that in the general population in two ways. First, as rifamycins interact with immunosuppressive drugs of the calcineurin inhibitor family (cyclosporine and tacrolimus), rapamycin and corticosteroids [90-93], rifamycin-sparing treatment regimens are preferred by many physicians. If a rifamycin is used, the risk of rejection may be increased due to lowered levels of calcineurin inhibitors; consequently, levels of cyclosporine or tacrolimus should be carefully monitored and doses should be adapted (3-5-fold increase) [93]. In cases of severe TB and/or if the organ is not vital, any effort to reduce immunosuppression should be undertaken. Interactions between first-line anti-TB drugs and immunosuppressive agents most commonly used in SOT recipients are summarised in table 3. Secondly, adverse anti-TB drug events are more frequent. Consequently, one or more first-line drugs cannot be used and thus the recommended duration of therapy is generally longer than in the general population $[94,95]$. The length of treatment and the drugs used after the first 2 months are controversial areas, especially if rifampicin is not used in the first 2 months or must be suspended due to intolerance. Recommendations given below are derived from studies in immunocompetent and other immunosuppressed individuals as direct evidence in transplant recipients is lacking. Daily dosage is recommended in all cases.

The standard short course anti-TB drug regimen, i.e. a 2-month initiation phase with a four drug combination of isoniazid, rifampicin, ethambutol and pyrazinamide, followed by a 4month continuation phase with isoniazid and rifampicin (2 $\mathrm{RHZE} / 4 \mathrm{RH})$, is recommended for most cases in the transplant setting, and particularly for severe and/or disseminated forms of TB. Based on expert opinion, the continuation phase should be extended to 7 months in patients with pulmonary TB and cavitation on the initial chest radiograph or if sputum cultures remain positive at 2 months of treatment as these patients have a higher rate of relapse [96]. Some experts recommend a continuation phase of 7-10 months in central nervous system (CNS) TB, mainly because of the increased risk of morbidity and mortality in these patients $[96,97]$.

Clinically stable patients with localised, non-severe forms of TB (excluding CNS, pericardial, osteoarticular and disseminated disease) and no suspicion or evidence of resistance to isoniazid may be treated with a rifamycin-free regimen, which will avoid drug interactions and may possibly reduce the risk of rejection [30]. However, results from a large case series of renal transplant recipients with $\mathrm{TB}$, of whom $>90 \%$ were treated with rifampicinbased regimens, indicate a similar rate of graft loss compared to the national mean [98]. Rifabutin interaction with immunosuppressive drugs is less important, and can be used instead of rifampicin with similar efficacy and possibly lower risk of graft loss. In rifamycin-free treatment regimens, combination therapy with isoniazid and ethambutol is recommended for 18 months with the addition of pyrazinamide for the first 2 months [97]. Irrespective of whether a rifamycin is part of an anti-TB drug regimen, the risk of disease recurrence is low when treatment is extended beyond 12 months [99]. Isoniazid-free and pyrazinamide-free regimens similar to those used in immunocompetent individuals $[96,100]$ should be used in case of resistance or intolerance.

Fluoroquinolones are an interesting drug class for anti-TB treatment, given that one or more first-line drugs cannot be used in many SOT patients. Evidence from immunocompetent individuals suggests that later generation fluoroquinolones (i.e. gatifloxacin or moxifloxacin and probably to a lesser extent also levofloxacin, but not ciprofloxacin or ofloxacin) can probably be used as alternative drugs to oral first-line agents [101]. Moxifloxacin has been shown to be equivalently effective to isoniazid in achieving culture conversion in the initial phase of treatment in one clinical trial [102]. There is also circumstantial evidence for the use of fluoroquinolones in SOT recipients. However the optimal duration of fluoroquinolone-containing regimens is unknown. Combined and prolonged use of levofloxacin and pyrazinamide has been associated with poor gastrointestinal tolerance [103, 104].

\section{Special situations}

In HIV-infected transplant recipients, rifamycins may lead to greater hepatotoxicity and jeopardise antiretroviral therapy because of their interaction with protease inhibitors and nonnucleoside reverse-transcriptase inhibitors. The recommended regimen in this population is isoniazid, pyrazinamide and ethambutol with moxifloxacin or levofloxacin [105].

In patients with significant hepatic disease and after liver transplantation, initial treatment with isoniazid, rifampicin and pyrazinamide in patients with TB has been associated with an increased risk of hepatotoxicity. Close monitoring of liver enzymes is necessary [106] and a pyrazinamide-free regimen is recommended in non-severe hepatic disease. In severe hepatic disease, an isoniazid- and pyrazinamide-free regimen (and even rifampicin-free) should be considered $[96,100]$. In severe hepatic disease or early after hepatic transplantation, a combination of ethambutol with a later generation fluoroquinolone (e.g moxifloxacin) might be a temporary solution until a more effective regimen can be administered.

Immune reconstitution inflammatory syndrome (IRIS) can occur in SOT recipients with TB under treatment [107]. A paradoxical worsening of symptoms with fever, cough, lymph node enlargement or roentgenographic abnormalities within the first 3 months of anti-TB treatment initiation has been described [108]. High-dose systemic corticosteroids are the most commonly employed treatment [109].

Macrophage activation syndrome (or haematophagocytic syndrome) is a relatively rare association in transplant recipients with opportunistic infections including TB [110]. The clinical picture combines fever, hepatosplenomegaly, pancytopenia and liver dysfunction, and histologically consists of bone marrow and organ infiltration with non-malignant macrophages phagocytising erythrocytes. Fatality is high $[98,110]$ and optimal treatment is unknown.

Multidrug resistant (MDR)-TB (i.e. resistance to isoniazid and rifampicin) has rarely been reported in SOT [111-113] or HSCT recipients [114]. Despite limitations in drug susceptibility testing (DST) MDR-TB should be treated according to the results of DST with at least four drugs that are active against $M$. tuberculosis in vitro. The selection of drugs for the treatment of MDR-TB should be performed in a hierarchical order. 
Extensively drug-resistant (XDR)-TB (i.e. MDR-TB plus in vitro drug resistance against any fluoroquinolone and one of the injectable aminoglycosides/polypeptides) has not yet been reported in transplant recipients and should, in case of occurrence, be treated following the same principles as for MDR-TB.

The World Health Organization (WHO) suggests a total duration of treatment of 18 months following $M$. tuberculosis culture conversion in MDR-/XDR-TB [115]. Validated biomarkers to guide physicians in the decision for treatment discontinuation are lacking. Adjunctive surgery may be required in selected patients [116]. When MDR-/XDR-TB is suspected, the decision for treatment initiation and the choice of anti-TB drugs should be made on an individual basis involving a physician with experience in the treatment of MDR-/XDR-TB.

\section{Other}

Adjunctive therapy and treatment monitoring for efficacy and adverse effects are similar as in immunocompetent individuals. In pulmonary TB, sputum smear and culture should be performed as a minimum at 2 and 4 months of treatment, at the end of treatment and on two further occasions until the end of a year. Extrapulmonary TB should be followed clinically.

Doses should be adjusted according to creatinine clearance. In case of hepatic disease, or elevated risk of drug-induced liver injury, frequent monitoring of serum aminotransferases and bilirubin should be performed and treatment regimens changed in case of a significant hepatotoxic reaction. At the beginning of a rifamycin-based regimen the dose of cyclosporine, tacrolimus or sirolimus should initially be increased three times when the rifamycin is introduced and then adjusted according to regularly measured serum levels. Similarly, when rifamycin is stopped, the dose of either immunosuppressive drug should be reduced to the pre-rifamycin dosage, and again adjusted by frequent serum level measurements. Oral corticosteroid dose should be increased by $50 \%$ during treatment with a rifamycin.

Isolation is particularly important in transplant recipients as they might come into contact with other transplant recipients in medical facilities. Pulmonary TB suspects should be isolated until active TB has been excluded. Pulmonary TB patients should be isolated for at least 2 weeks if $M$. tuberculosis strains are sensitive; many centres advocate isolation until they have three consecutive negative sputum smear results. Continuous isolation until $M$. tuberculosis cultures are negative may be advocated if patients are in contact with other immunocompromised individuals although the risk of transmission is low in patients with sputum conversion on treatment.

\section{Summary}

1. In general, the same short-course treatment regimen (2 $\mathrm{HRZE} / 4 \mathrm{RH}$ ) is recommended for transplant recipients as for other patients with TB.

2. TB treatment in transplant recipients is often complicated by interactions between rifamycins and immunosuppressive drugs and the increased frequency of adverse anti-TB drug events.

3. A rifamycin-free anti-TB treatment regimen is an important option in non-severe cases in order to avoid drug interaction with immunosuppressive drugs and thus reduce the risk of graft rejection.

4. Fluoroquinolones are promising drugs in the treatment of TB in transplant recipients although clinical evidence for the treatment efficacy are still lacking.

5. Length of continuation phase is dependent on the drugs used in the initial phase and in the continuation phase; except for the standard regimen, many recommendations are extrapolated from immunocompetent individuals or are opinion based.

\section{SPECIAL CONSIDERATIONS IN STEM CELL TRANSPLANT RECIPIENTS}

As with SOT patients, the frequency of TB in HSCT recipients expectedly correlates with the local notification rates of incident TB. Areas where TB incidence is high report a TB frequency of $1-16 \%$ during follow-up [117-120]. In developed regions, the reported proportions range from 0.4 to $2.2 \%$ [121-123]. Published cases from developed nations commonly include immigrants from countries where TB is endemic, other groups with increased risk and thus prevalence of infection, prisoners or contacts with a history of exposure [123]. In the USA, the frequency of TB in HSCT is approximately double that in the general population, underscoring the effects of immunosuppression [5]. Nevertheless, TB is more frequently diagnosed in SOT than in HSCT recipients [124]. This disparity may reflect the immune reconstitution in HSCT, notably of cellular immunity, and/or the impact of the use of fluoroquinolones in antibacterial prophylaxis during neutropenia with incidental anti-mycobacterial efficacy $[119,124]$.

TB has generally been observed as a delayed complication of HSCT occurring beyond day 100. Early presentations have been reported, often as a cryptic source of fever after HSCT [125]. Cases are predominantly pulmonary with variable radiographic features, and dissemination is documented infrequently. Experience in Hong Kong and Taiwan document upper lobe-predominant disease suggestive of reactivation TB $[119,126]$. In a South Korean series, nodular or multi-lobar airspace disease was more common without zonal predominance, consistent with primary TB [127].

Prospective donors with symptoms of TB should be evaluated for active disease and should not donate until TB has been adequately treated. Routine screening for active TB of potential donors from endemic regions for TB has not been studied, but appears to be unnecessary.

\section{Risk factors}

Recipients of allogeneic stem cell grafts, particularly matched unrelated grafts, appear to be at higher risk for $M$. tuberculosis infection than recipients of autologous stem cell grafts [124-126]. In autologous transplantation, risk factors for mycobacteriosis due to environmental mycobacteria include conditioning regimens affecting T-cell function such as fludarabine, antithymocyte globulin (ATG), or alemtuzumab [128]. In some series, development of TB was found to be related to receipt of T-cell depleted allografts [129]. Administration of corticosteroids was significantly associated with development of TB in one case series [124]. Conditioning with total body irradiation has also been cited as another risk factor [117, 119, 124]. In multiple series, acute or chronic graft versus host disease (GvHD) with associated depression of phagocytic and cellular immune 
functions is the most strongly associated risk factor for the development of TB $[119,125,126,129]$. Following $M$. bovis BCG vaccination, transfer of PPD-reactive memory $\mathrm{T}$-cells from donor to recipients is not protective in individuals suffering GvHD [130].

No correlation has been found between specific conditioning chemotherapeutic regimens or GvHD prophylactic regimens and the risk for TB. Reactivation of cytomegalovirus has been unreliably reported in HSCT patients who contract TB, precluding analysis of correlation. New biologic agents (TNF antagonist therapies, T- and B-lymphocyte depletion or co-stimulatory blockade) are additional risk factors for TB. These agents can be used either as a part of the conditioning before HSCT or as a treatment for GvHD, a condition associated with increased risk for TB. There are case reports of TB in patients given alemtuzumab as part of the conditioning therapy [131], and one suspected but unproven case of TB associated with rituximab given for chronic GvHD [132]. Although there are few data in HSCT, most of these agents have been associated with an increased risk for TB in other patient populations and may pose an increased risk in the HSCT population as well $[133,134]$.

\section{Prevention, diagnosis and therapy}

Evaluation for LTBI or TB should be performed in candidates for HSCT who have a history of prior exposure to TB [135]. Screening should be performed in recipients from endemic regions using TST or IGRA. Prior chemotherapy may reduce TST responses [136]. IGRAs may be useful in screening patients with prior $M$. bovis BCG vaccination [136, 137]. Use of IGRAs for LTBI in HSCT patients is under investigation. Available data suggest a high percentage of indeterminate test results, especially in the first year after transplantation [59]. Any individual with a positive $M$. tuberculosis-specific immunodiagnostic test should be evaluated for active disease.

When identified and treated before HSCT, TB rarely recurs $[117,124]$. No standardised screening protocol exists for LTBI prior to HSCT. A multicentre study of 351 allogeneic stem cell recipients screened by chest radiograph prior to HSCT from Turkey examined pre-HSCT screening strategies: 1) chest radiography only; 2) use of PPD with isoniazid therapy for TST $>15 \mathrm{~mm}$; or 3) universal use of INH prophylaxis immediately prior to and for 6 months following receipt of HSCT [118]. No TB occurred after HSCT in patients given isoniazid either by mandatory protocol or for a positive PPD. None of seven patients who had a positive PPD pre-HSCT with a negative chest radiograph developed TB. In the series, five out of 274 untreated patients developed pulmonary TB. Among these five patients, all had clear chest radiographs, two had not been tested by PPD and three had a TST $<10 \mathrm{~mm}$ prior to HSCT.

In general, it is reasonable in endemic regions to provide empiric prophylaxis based on either exposures or screening test positivity. The interpretation of TST results may be difficult, as the underlying disease or prior chemotherapy may lead to skin test anergy. Such individuals merit careful follow-up. This also underscores the need for more accurate diagnostics in this population. Live $M$. bovis BCG vaccination is contraindicated in HSCT recipients [138, 139].

As with SOT patients, diagnosis of TB is often delayed in HSCT recipients given the non-specific signs and symptoms of $\mathrm{TB}$, often compounded by a low index of suspicion in low-incidence countries. The diagnosis of all forms of TB should be based on microbiologic evaluation, including antimicrobial susceptibility data on the isolates, whenever possible. Initial therapy may be empiric and based on microscopic demonstration of acid-fast bacilli, clinical suspicion, radiographic suggestive presentation or histology; therapy should not be delayed until culture results are available if other evidence is sufficiently strong to warrant intervention [126]. The use of nucleic acid amplification or hybridisation techniques has been incompletely evaluated in this population.

The mortality of patients with TB after HSCT is reported to range between 0 and 50\% [7]. TB following autologous transplantation generally responds well to treatment [124, 125]. To date, few cases of MDR-TB have been reported in the HSCT population $[119,120]$.

\section{Summary}

1. The magnitude of the TB risk in HSCT recipients is strongly associated with the epidemiology of TB in the general population to which the patients belong. Recipients of allogeneic HSCT, particularly matched unrelated grafts, are at higher risk of TB than recipients of autologous HSCT.

2. The risk of death from or with TB after HSCT is high (up to $50 \%$ ).

3. Evaluation for LTBI or TB should be performed in candidates for HSCT with possible increased exposure risk. Screening should be performed in recipients from endemic regions using TST or IGRA. Prior chemotherapy may reduce TST responses.

4. TB after HSCT is predominantly pulmonary with a wide spectrum of radiographic features. TB is generally a late complication of HSCT occurring beyond day 100. Earlier disease manifestations often include presentations as cryptic febrile illnesses.

5. Acute or GvHD is an independent risk factor for the development of $\mathrm{TB}$ in addition to the intensity of immunosuppression.

\section{SPECIAL CONSIDERATIONS IN CHILDREN}

\section{Epidemiology of TB in paediatric transplant recipients}

Data regarding TB in paediatric transplant recipients are scarce; many studies do not include children or results are not reported separately from adult data. However, published reports of small numbers of cases from single institutions suggest that rates of TB following liver transplantation in children range from $2.4 \%$ in non-endemic areas to $3.6 \%$ in highly endemic areas [140, 141] while rates following renal transplantation of $8-9.7 \%$ have been reported from highly endemic areas [142, 143]. In agreement with reports in adults, the incidence of TB following bone marrow transplantation is lower than that observed with SOT, with rates of $1.7 \%$ reported in a retrospective study in India [144]. Whilst published estimates of the incidence of TB are lacking, these data suggest that rates of TB in paediatric transplant recipients are significantly higher than in the general population, in common with the increased risk observed in adult recipients [54]. Incidence of TB varies with the type of organ transplanted and population prevalence of TB. 


\section{Paediatric TB}

Paediatric TB is most commonly a result of primary infection and therefore a previous history of TB is infrequently found in children who develop the disease following transplant $[141,145]$. TB arising from the donated organ would be expected to be uncommon [76].

Children have an age-dependent increased risk of progression from LTBI to disease; those aged $<5$ yrs, adolescents and young adults have the highest risk of disease and school-aged children have the lowest risk [146]. Immunosuppression further increases the risk. Children, particularly of very young age, who develop $\mathrm{TB}$ are more prone to present with extrapulmonary and disseminated forms compared to adults, a risk that is also reflected in the presence of immunosuppression [147]. Whilst the majority of reported cases of TB in paediatric transplant recipients are pulmonary, some case series report frequencies of disseminated disease and extrapulmonary disease in excess of 50\% [141-144, 148]. Compared to the adult population, children who develop TB have an increased risk of death. One case series reports mortality of $>30 \%$ in paediatric transplant patients who develop TB [141].

\section{Diagnosis of infection with M. tuberculosis}

Depending of the age of the child, the source of infection is commonly an infectious adult living in the same household, making screening of family members for active disease important in paediatric transplant candidates [146]. In one study, $80 \%$ of children who developed TB following liver transplantation had a family member identified as having TB or $M$. tuberculosis infection.

Screening of children for infection with $M$. tuberculosis relies on history of exposure, TST and the exclusion of clinical and radiological signs and symptoms of tuberculosis. IGRAs have recently been included in national guidelines in some parts of Europe $[97,149,150]$. TST can be influenced by recent $M$. bovis BCG vaccination and environmental mycobacteria and it may be falsely negative in young children and those who are immunosuppressed. In support of this, in one UK case series the TST was unreactive in five out of six children who developed TB following liver transplantation [141]. IGRAs were not used in this study. Whilst neither M. bovis BCG nor most environmental mycobacteria influence the result of IGRAs, data regarding their value in immunosuppressed children and young children are lacking. Studies evaluating IGRAs in transplant candidates to date have excluded children [62, 63].

The diagnosis of active TB is similarly challenging in children due to the difficulties collecting specimens, the paucibacillary nature of disease and the increased possibility of non-specific symptoms and signs. Diagnosis in paediatric transplant recipients may be even more challenging as these difficulties may be compounded by immunosuppression and necessitate invasive sampling methods [141, 148]. Recent advances in TB diagnostics may assist in the diagnosis in this vulnerable population; however, they urgently require evaluation in children [151].

\section{Prevention of TB in paediatric transplant recipients}

Identification and treatment of household members with active disease is important in the prevention of $\mathrm{TB}$ in paediatric transplant candidates and recipients. Although immunosuppressive therapy might impair performance of both TST and IGRA, it would be prudent to screen children prior to transplantation with these tests. If they are positive, active TB needs to be excluded and if they are negative this might indicate genuine absence of $M$. tuberculosis sensitisation or false-negative results. In children identified as having LTBI, one should consider preventive therapy with 9 months of isoniazid, commencing prior to transplantation. However, there are concerns regarding the liver toxicity of isoniazid preventive therapy in paediatric transplant candidates and recipients. VERMA et al. [141] report that two out of six children treated for TB with isoniazid developed isoniazid-induced hepatitis, although this was resolved with dose reduction. The lack of data makes recommendations difficult; some institutions recommend use of isoniazid preventive chemotherapy, whilst others withhold it or reserve preventive treatment for children at highest risk only [140-142].

\section{Summary}

1. Few studies report the incidence of $\mathrm{TB}$ in paediatric transplant recipients and the numbers of children in such studies are very small; however, available data are consistent with that reported in adult transplant recipients.

2. There are a number of features unique to paediatric TB which are likely to be amplified in children who are immunosuppressed following transplantation, namely an increased risk of progression from infection to disease, extrapulmonary, including disseminated disease, and higher risk of death from or with TB.

3. Screening of paediatric transplant candidates for M. tuberculosis infection and screening of family members for active disease is important and careful consideration must be given to the provision of isoniazid preventive therapy to those identified as having LTBI to decrease the TB risk following transplantation.

4. Whether TST or IGRA or both are the preferred approach remains unresolved, and which strategy is being used will also depend on resource availability.

\section{PUBLISHED NATIONAL GUIDELINES}

Guidance on assessing and managing both LTBI and TB in SOT or HSCT recipients has been produced in several countries. Considering only English language publications, seven national guidelines have been identified [4, 30, 138, 152-155], six of which relate to SOT and one to HSCT. Some offer guidance on screening of living and deceased donors in addition to screening and management of LTBI and TB in the recipients. These publications are from the following countries or geographic areas: Canada [152], Europe [153], Spain [30], UK [154], USA [4, 155] or global [138]. Four deal specifically with renal transplantation [4, 152-154], two with SOT in general $[4,30]$, including some specific information on liver and lung transplants, and one with HSCT [138]. In addition, there are four further statements relating to TB and transplantation: one on general testing and treatment of LTBI [156]; a conference update briefly covering cardiac transplantation [157]; a general statement [158]; and a review of the evidence for preventive chemotherapy/chemoprophylaxis [31].

The guidelines and statements are hitherto referred to as "guidelines" and are discussed and compared on screening for LTBI and 
$\mathrm{TB}$, recommendations for preventive chemotherapy/chemoprophylaxis and regimens, and therapy for TB and its duration.

\section{Points of agreement}

There is general consistency in most of the recommendations made across all the publications. Some are more comprehensive than others. Where mentioned, all guidelines recommend screening for evidence of LTBI and TB, including taking a full clinical history and making a physical examination as part of the initial assessment, with the exception of the HSCT guidelines [138] which recommend screening only for demonstrable risk. The breadth of recommended history taking varies but includes details on ethnicity, country of birth, history of recent contact with TB, previous disease and treatment, together with evaluation for any additional risk, such as diabetes or travel to countries of high background incidence. A chest radiograph is recommended, to be supplemented by a TST and/or an IGRA by all guidelines. The more recent guidelines [4, 138, 154-156] include IGRA testing, with or without TST. All suggest screening pre-transplant, preventive therapy for LTBI with isoniazid plus pyridoxine (vitamin B6), monitoring of liver enzymes and full treatment for active TB.

\section{Areas of difference}

The UK and HSCT guidelines do not give a cut-off point for a positive TST as they make the point that the TST is unreliable in patients with advanced chronic kidney disease or who receive immunosuppressive treatment. A positive test is judged to be useful but a negative test is not because of too frequent falsenegative results. The UK guidelines [154] recommend using an IGRA with or without a TST. The HSCT guidelines [138] consider an IGRA to be more useful in the immunocompromised but note that a negative test does not exclude LTBI, a point also made by the US renal guidelines [155]. Most of the other guidelines recommend a TST cut-off of $\geqslant 5 \mathrm{~mm}$ as a positive result, but the European guidelines draw a distinction between transplant recipients $(\geqslant 5 \mathrm{~mm})$ and dialysis patients $(\geqslant 10 \mathrm{~mm})$. The Spanish [30] and US [4] guidelines recommend repeating the TST to increase sensitivity by provoking a "booster effect".

In general, all include isoniazid as single agent, most recommending 9 months of treatment, with the exception of the UK [154] where 6 months is recommended as "regimens longer than 6 months isoniazid have only very minimal additional advantage at the cost of an increase in the risk of hepatitis" [159]. The European and US SOT guidelines $[4,153]$ recommend 9 months of isoniazid but include a caveat that this can be shortened to 6 months. Other regimens suggested by different guidelines include 3 months isoniazid plus rifampicin, 4-6 months rifampicin alone, and 2 months rifampicin plus pyrazinamide (table 4).

The UK guidelines [154] are unique in attempting to quantify the relative risk of reactivating TB compared with the risk of developing significant hepatitis resulting from various regimens. As neither the TST nor the IGRAs are wholly reliable indicators of LTBI, this can aid the clinical decision on which patients should receive preventive chemotherapy/chemoprophylaxis.

Most guidelines recommend standard quadruple therapy for active TB for a total of 6 months for non-CNS TB that is fully drug susceptible. Although all raise the issue of drug interactions between rifampicin and immunosuppressive drugs (table 3), the
Spanish guidelines [30] are alone in recommending avoiding rifamycins, except in disseminated disease and where isoniazid resistance is a possibility. All recommend dose monitoring of immunosuppressive drug levels and the UK guidelines [154] recommend doubling the dose of corticosteroids in patients taking rifampicin. Rifabutin is frequently suggested as an alternative to rifampicin as it is a less potent inducer of cytochrome P450. A summary of the main recommendations made in the different publications can be found in table 4 .

\section{Summary}

1. There is overall agreement in most recommendations found in seven national, regional, and global guidelines together with three further relevant short statements relating to screening for LTBI and active TB.

2. Preventive therapy with isoniazid, isoniazid plus rifampicin or rifampicin alone is generally recommended pre-transplant if there is evidence of LTBI or chemoprophylaxis if there is a significant risk.

3. Standard treatment is recommended for active TB with some guidelines recommending prolonged treatment.

4. Attention should be paid to serum levels of immunosuppressive drugs when rifampicin is used.

\section{CONSENSUS RECOMMENDATIONS}

Current evidence regarding optimal management for the prevention and treatment of $\mathrm{TB}$ in transplant recipients is limited. Recommendations made in this consensus statement largely rest on expert opinion and inference derived from TB prevention and treatment in the non-transplant setting (table 1). These consensus recommendations aim to guide physicians responsible for the care of transplant recipients until better clinical evidence becomes available.

\section{Is screening for latent infection with M. tuberculosis recommended for all transplant candidates?}

The indication for screening should be guided by locally established frequency of TB in transplant recipients. Preventive chemotherapy against TB without prior screening for LTBI may be appropriate for all transplant recipients in regions of high TB incidence ( $\geqslant 100$ per 100,000 population; evidence level D). In regions of medium TB incidence ( $\geqslant 20$ per 100,000 population or in regions with medium local TB burden), all candidates should be screened for the presence of $M$. tuberculosis specific immune responses (the best available proxy for LTBI; evidence level C). In regions of low TB incidence, a decision for screening of transplant recipients for the presence of $M$. tuberculosis specific immune responses should include an individual risk assessment for LTBI. In centres where LTBI screening is not performed for all transplant recipient candidates as a routine procedure, screening should be performed in candidates with at least one additional risk factor for infection with $M$. tuberculosis, e.g. a recent contact to an infectious patient with $\mathrm{TB}$, immigration from a high incidence country of $\mathrm{TB}$, a medical history of previous untreated or insufficiently treated TB or fibrotic/calcified lesions on chest radiography compatible with a past history of TB in previously untreated patients (evidence level D). All children should be screened (evidence level D).

In general, all transplant candidates should be questioned about a history of prior TB or TB contact (including a past TST or IGRA) 


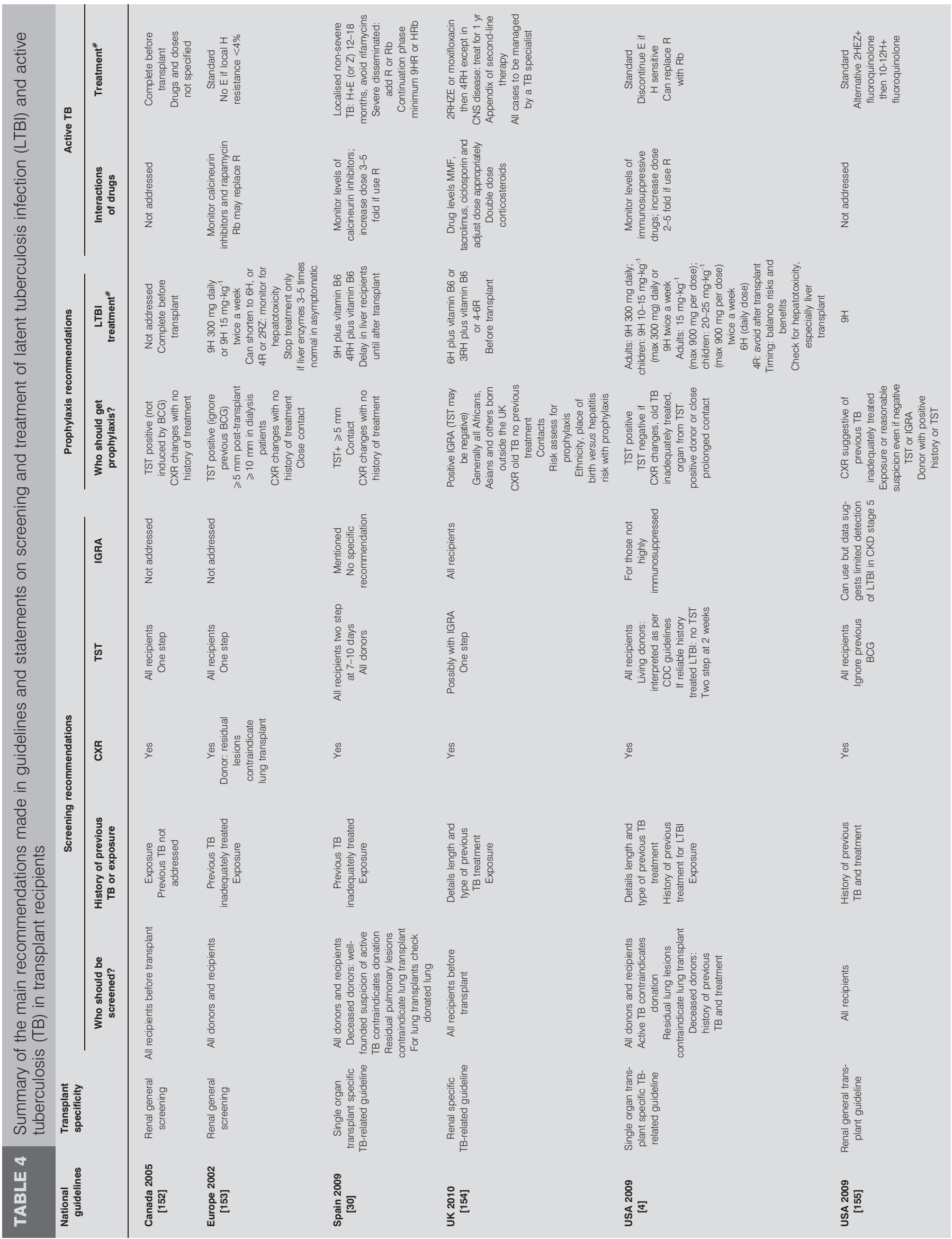




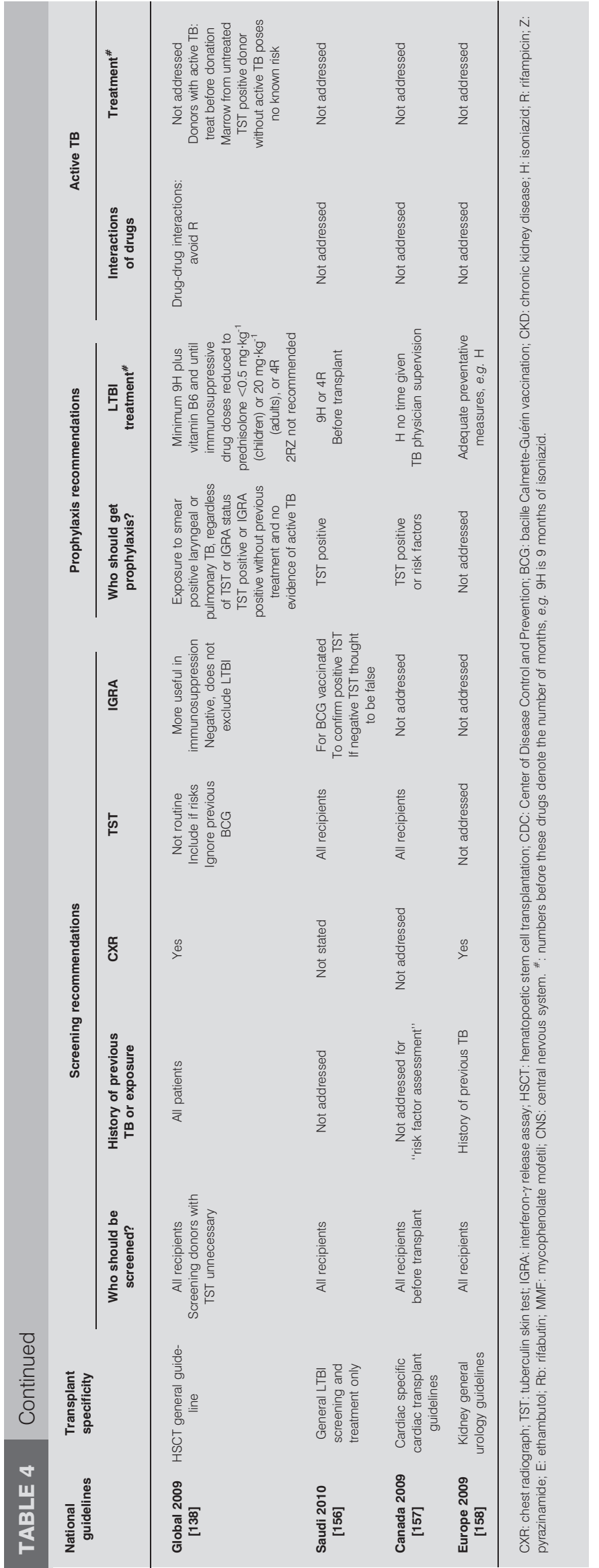

and should have a chest radiograph to search for evidence of prior or current TB. In case of abnormal radiographic findings compatible with previous (such as fibrotic lesions, calcified granulomas or lymph nodes, pleural thickening with or without calcification) or active TB (fibronodular or cavitating lesions), an additional CT scan may provide more specific information (evidence level B).

Is there a difference in the recommendations for screening of recipients of solid organs or hematopoietic stem cells? The same recommendations apply (evidence level D). However, the risk of false-negative immune responses is considerable in persons already using immunosuppressive medication or following recent chemotherapy for haematological malignancy, or in chronic renal failure.

\section{Should screening for latent infection with M. tuberculosis be performed by skin testing, IGRA, or both?}

The best choice for a screening test to detect LTBI in candidates awaiting SOT or HSCT is unclear, as the positive predictive value for the development of $\mathrm{TB}$ in this setting is largely unknown and varies with TB prevalence. In general, neither IGRAs nor TST have high accuracy for the prediction of active TB (evidence level B). Until more data on the predictive ability are available, the choice of the test should be based on the relative specificity in different prevalence regions, logistics and cost. IGRAs have some operational advantages as mitogen controls may allow differentiation between anergic and nonsensitised individuals. TST should be interpreted carefully in individuals with a known history of $M$. bovis BCG vaccination, although the effect of such vaccination wanes after one to two decades; IGRAs can help distinguish responses due to BCG vaccination from infection with $M$. tuberculosis. Given the discordant results between IGRAs and TST in several studies in immunocompromised hosts, a combination of both assays may result in a higher sensitivity if false negativity is a concern. This approach may be particularly useful in children. It is currently unclear, however, whether the combination will result in a better risk estimation for the development of TB after transplantation. For the diagnosis of latent infection with $M$. tuberculosis in low prevalence settings, experts suggest using the QuantiFERON ${ }_{\AA}$ TB Gold in-tube test or the T-SPOT.TB® test (evidence level D). If IGRAs are not available, TST should be used for screening for LTBI. A higher risk of false-negative immunological test results in patients with recent chemotherapy or T-cell depleting agents should be taken into account.

If IGRAs are used for the diagnosis of latent infection with M. tuberculosis, is there a preference for QuantiFERON $N_{\mathbb{R}}$ TB Gold in-tube or T-SPOT.TB ${ }_{\circledast}$ test?

There is no evidence available to favour either test for diagnosing LTBI in transplant candidates. There is some evidence of increased sensitivity of the T-SPOT.TB $B_{\circledast}$ test over QuantiFERON ${ }_{\circledast}$ TB Gold in-tube test in HIV-infected individuals with low CD4 counts (evidence level C).

Which cut-offs should be used for the TST and IGRAs for the diagnosis of latent infection with $\mathrm{M}$. tuberculosis in transplant candidates?

Pending further evidence, the cut-offs for IGRAs for the diagnosis of LTBI in candidate recipients of SOT or HSCT should be used as 
recommended by the manufacturers for Europe (evidence level D). A TST result of $\geqslant 10 \mathrm{~mm}$ is commonly used as being indicative of LTBI in the non-BCG vaccinated individual and should not generally need confirmation by an IGRA. A TST result of $\geqslant 5$ and $<10 \mathrm{~mm}$ in a person using immunosuppression may be considered as probable LTBI in the absence of BCG vaccination, but specificity at such low cut-off points is often poor due to the possibility of sensitisation with environmental mycobacteria, while the gain in sensitivity might be marginal [40].

In children, a cut off of $\geqslant 5 \mathrm{~mm}$ is recommended as it is assumed that accumulated non-specific cross-reactions are less frequent; however, the influence of BCG, where given at birth, might be considerable. TST and IGRA should both be performed at the same time. If the result of the IGRA is positive, the child should be treated for LTBI once active TB is excluded. If the result of the IGRA is negative and the TST induration is $\geqslant 10 \mathrm{~mm}$ in an immunocompetent child or $\geqslant 5 \mathrm{~mm}$ in an immunocompromised child, a diagnosis of LTBI should be assumed (evidence level D).

\section{What decision should be taken when testing for latent infection with $\mathrm{M}$. tuberculosis provides a positive test result?}

All candidates with a positive TST or IGRA test result after targeted screening should receive preventive chemotherapy to reduce the risk for the development of TB ("intention to test is intention to treat"; evidence level A).

\section{What decision should be taken when testing for latent infection yields discordant results (e.g. positive TST and negative (GRA)?}

If results from both tests are available and are discordant, then the positive result, be it IGRA or TST, should be used for clinical decision making regarding preventive chemotherapy (evidence level D). This should also be the approach taken in children, whether BCG vaccinated or not. The reason for accepting a positive test as a basis of therapy is that false-negative results for either test are common; moreover, it is not possible to disprove that a positive test result is truly positive. In individuals with a history of $M$. bovis BCG vaccination in the last $10 \mathrm{yrs}$ and a positive TST result of $<15 \mathrm{~mm}$ ( $<10 \mathrm{~mm}$ if BCG vaccination was performed $>10$ yrs ago), with a normal chest radiograph, and in the absence of additional risk factors, TST may be regarded as a false-positive. The risks of withholding preventive therapy should be discussed with the patient and the final decision should be agreed upon by all parties involved. Posttransplantation vigilance should be high in this situation with a low threshold for further diagnostics.

\section{Under which circumstances should preventive chemotherapy against TB be offered to transplant candidates in the absence of immunodiagnostic testing or in the presence of negative results of immunodiagnostic testing?}

In the absence of immunodiagnostic testing or in the presence of a negative result of immunodiagnostic testing, treatment for the prevention of TB should be offered to: all individuals with fibrotic or calcified lesions on chest imaging as putative radiographic evidence of previous TB who did not receive adequate anti-TB chemotherapy; individuals with a strong history of exposure or even documentation of a previous positive TST or IGRA result; individuals originating from a country with a very high incidence (e.g. $\geqslant 100$ per 100,000 population per year); and any child with recent documented exposure to bacteriologically confirmed TB (evidence level C).

\section{When should infection with M. tuberculosis be expected in a donor graft?}

Infection with $M$. tuberculosis of the donor graft is likely to be related to the risk of $M$. tuberculosis infection of the donor and the type of graft. All organs of donors with active or inadequately treated TB should be considered infectious for the immunocompromised recipient. In lung transplantation, lungs from donors with evidence for LTBI (based on a prior positive TST or prior/actual IGRA result in the absence of active TB) should be considered as potentially infectious. To what extent transplantation of organs other than lungs from donors with LTBI increases the risk for TB in the immunocompromised recipient is unknown. However, organs derived from individuals with likely $M$. tuberculosis exposure in endemic regions may potentially contain live M. tuberculosis (evidence level D).

\section{Should all transplant candidates from a donor who is/was thought to be infected with $\mathrm{M}$. tuberculosis be offered prophylactic treatment against TB?}

Active TB in a SOT donor should be assessed and if positive excluded from donation (evidence level C). Haematopoietic stem cells are highly unlikely to harbour live $M$. tuberculosis except perhaps in a person with disseminated TB at the time of harvest (evidence level D). Solid organs from donors with LTBI may contain live M. tuberculosis, especially the lungs, but transmission via other organs has only been described in rare instances. For living donors, the benefit of prophylactic chemotherapy to reduce the risk of $M$. tuberculosis transmission is uncertain. If LTBI is thought to be present in a deceased donor, the recipient of the lung transplant should be treated for LTBI while the benefit in recipients of other organs may be outweighed by potential toxicities (evidence level D). Clinical monitoring and constant vigilance is mandatory in the posttransplantation period.

\section{Which regimen for preventive chemotherapy/ chemoprophylaxis is most effective in reducing the risk of developing TB in recipients who either have positive $\mathrm{M}$. tuberculosis-specific immune responses or receive a graft from an individual that is thought to be infected with $\mathrm{M}$. tuberculosis?}

Few data exist to use in the selection of specific regimens based on factors other than drug toxicity and interactions. In endemic regions, treatment of individuals with preventive chemotherapy/chemoprophylaxis with positive screening assays prior to immunosuppression results in a reduced rate of clinical TB. However, the effectiveness of various chemotherapeutic regimens to prevent TB has not been studied in this setting. The best clinical evidence of efficacy in other populations supports a choice of a preventive chemotherapy regimen of 9-12 months of isoniazid (evidence level A). The efficacy of that regimen exceeds $90 \%$ and the effectiveness exceeds $60 \%$. An alternative choice of 4 months of rifampicin or a combination of 3 months isoniazid plus rifampicin or rifapentine cannot be routinely recommended for the transplant setting due to interactions of immunosuppressive drugs with rifamycins, 
but can be considered if pre-transplant treatment is possible (evidence level B). The interaction between rifampicin and anticoagulant or other drugs such as $\beta$-blocking agents can often be managed with dose adjustments and close supervision (evidence level C).

\section{When should transplantation be performed in relation to the initiation of preventive treatment of LTBI?}

If treatment of LTBI cannot be completed before transplantation, it should be completed after transplantation as scheduled, if possible (evidence level D). Given interactions with calcineurin inhibitors, rifampicin-free regimens may be preferred. Transplant patients are monitored closely during the first year, which will allow timely detection of TB reactivation. No specific additional diagnostic tests can be advised routinely, but new unexplained symptoms or signs should be analysed without delay. In this setting, the diagnostic approach must be aimed at direct detection of bacilli, and immunodiagnostic tests are not useful (evidence level C).

\section{What is the optimal treatment for active TB in transplant recipients?}

All treatments of active TB should be based on susceptibility testing of mycobacterial isolates. While awaiting such data, a standard daily regimen with a 2-month initiation phase with a four-drug combination HRZE followed by a 4-month continuation phase with $\mathrm{H}$ and $\mathrm{R}$ is recommended for recipients of SOT or HSCT (evidence level B). The continuation phase should be extended to 7 months in patients with pulmonary TB and both cavitation on the initial chest radiograph and/or positive sputum cultures at 2 months of treatment as these patients have a higher risk of relapse (evidence level D). Some experts recommend a continuation phase of 7-10 months in CNS TB mainly because rifampicin and ethambutol have reduced CNS penetration. If a rifamycin is used, the risk of rejection may be increased due to lowered levels of prednisone or calcineurin inhibitors; consequently, steroid dosage should be increased by $\sim 50 \%$, and levels of cyclosporine, tacrolimus and rapamycin should be carefully monitored and dosage increased accordingly. A rifamycin-free regimen $(\mathrm{H}, \mathrm{Z}, \mathrm{E}$ or fluoroquinolone) is an option in non-severe TB cases in order to avoid interaction with immunosuppressive drugs (evidence level C).

\section{How should patients who started preventive chemotherapy for LTBI be followed in relation to the timing of SOT or HSCT?}

Patients undergoing treatment for LTBI should be followed up with weekly assessment initially for liver enzyme testing and, if appropriate, immunosuppressive drug levels. If stable for 1 month, monthly assessments are likely to be adequate, including questioning about adverse effects of therapy and liver function testing. In case of symptoms, patients should be seen without delay to allow timely detection of adverse drug events or breakthrough TB (evidence level D).

\section{How should patients who started treatment of active TB be} followed in relation to the timing of SOT or HSCT?

Active TB under treatment is regarded as a relative contraindication for transplantation in many centres other than emergency heart, lung or liver transplantation. Individuals developing severe anti-TB drug-induced liver injury may also require emergency transplantation. In general, patients should have at least completed the induction period (2 months) and it is preferred, although not always possible, to complete the full treatment against TB prior to transplantation (evidence level D).

The treatment of TB in transplant patients is complex and requires a multidisciplinary approach. Frequent monitoring is required for early diagnosis of adverse events (including liver function tests) and for adjustment of immunosuppressive drug dosage. When potentially hepatotoxic drugs such as $\mathrm{H}, \mathrm{R}$ or $\mathrm{Z}$ must be avoided, MDR-TB treatment regimens may be an alternative. However, although evidence is lacking, recommended treatment durations for these regimens are up to 2 years following $M$. tuberculosis culture conversion (evidence level D).

\section{FUTURE RESEARCH DIRECTIONS}

Research directions should address both diagnosis and therapy of TB to improve TB management in transplant recipients.

\section{Improvements in the diagnosis of active TB}

Novel generations of automated nucleic acid amplification tests, e.g. the Xpert MTB/RIF test (Cepheid, Sunnyvale, CA, USA), could potentially improve the rapid diagnosis of TB in transplant patients. In immunocompetent patients the Xpert MTB/RIF test has a very high diagnostic accuracy; especially in patients with acid-fast bacilli, sputum-smear positive pulmonary TB [160, 161]. However, the diagnostic sensitivity for pulmonary TB is substantially reduced in immunocompromised patients with HIV-infection and acid-fast bacilli, sputumsmear negative pulmonary TB [162] and the value of this method for the rapid diagnosis of TB in transplant patients still needs to be established.

\section{Improvements in the diagnosis of LTBI in transplant candidates and donors}

Although the predictive value of IGRAs and the TST for progression to active disease have been studied in immunocompetent subjects $[46,69,70]$ its value to predict active TB after transplantation, the impact of various immune suppression regimens and pathogen prevalence have not been assessed [163]. Current generations of immunodiagnostic tests are poor predictors of future TB risk because of relatively low specificity for live bacilli in immunocompetent individuals, e.g. donors. In the immunocompromised host, such as transplant recipients, the concern is less with test specificity, but the recognised deficiency is test sensitivity. Unlike TST, IGRAs may have potential to be used for LTBI screening of deceased donors to improve donor-derived risk assessment, but this has not been formally tested. New diagnostic approaches are needed that allow for a more targeted identification of patients at risk to develop TB. This may involve modifications of in vitro immunodiagnostic assays such as the use of novel stimulatory antigens [15, 164, 165], alternative biomarkers other than IFN- $\gamma$ [166-172], variations in incubation time $[173,174]$, the readout system $[9,12,13$, $175,176]$ or the clinical specimen instead of blood [177-179]. In addition, both for the detection of LTBI with a risk of reactivation and for suspected active TB, a novel approach based on a whole blood transcriptional signature could provide a biomarker system with high discriminative potential [180]. While the results of this research may lead to an improvement of the current immunodiagnostic assays in general, it may be of 
particular value in immunocompromised patients including transplant recipients.

\section{Improvements on therapy}

Future clinical trials should separately address therapies in transplant candidates and in post-transplant recipients who are receiving immunosuppressive therapy. Potential improvement could be obtained by new drug combinations including novel drugs such as the diarylquinoline TMC207 [181], or combinations with immunopotentiators [182, 183]. The availability of treatment regimens with equal or better effectiveness that require shorter treatment durations and have fewer interactions with immunosuppressive drugs compared to current anti-TB therapies are desirable for both active disease and LTBI. In both situations, the optimal choice of a rifamycin is unclear as it needs to be established which regimens without rifamycins are most effective. Recently, a 3-month regimen including isoniazid and rifapentine once a week has been shown to be equally effective to 9 months daily isoniazid therapy in the prevention of TB in healthy contacts [184]. This drug regimen needs to be evaluated in the transplant setting. Special attention should be given to liver transplant recipients that may suffer from increased drug-mediated liver toxicity.

\section{Summary}

Research needs comprise the following areas: 1) the use of rapid nucleic acid amplification tests in the diagnosis of active TB in immunocompromised patients; 2) the use of IGRAs in deceased donors and the need for new diagnostic approaches for a more targeted identification of transplant candidates and recipients at risk of developing $\mathrm{TB}$; and 3) treatment regimens that require shorter treatment durations and have fewer interactions with immunosuppressive drugs.

\section{CONCLUSIONS}

Post-transplant TB is a rare complication after SOT or HSCT. However, it has a high morbidity and mortality and its treatment is complicated by several interactions between immunosuppressive and anti-bacterial drugs. Although TB occurs more frequently in transplant recipients compared to the general population, the management of post-transplant TB and widespread adherence to uniform guidelines is complicated by the fact that its overall prevalence varies considerably and largely depends on the local TB prevalence of a given geographic region. Hence, careful risk-adapted screening and preventive chemotherapy, as well as constant vigilance for early signs or symptoms of disease including prompt treatment, are currently the most effective modalities for prevention and management of TB post-transplant.

In this statement by the TBNET we have summarised the current knowledge of the risk of TB after SOT and HSCT and we have provided detailed consensus recommendations for the most important clinical questions related to its management in adults and children. Targeted TB screening adapted to the local or individual risk of latent $M$. tuberculosis infection and preventive chemotherapy for all individuals with evidence for latent infection with $M$. tuberculosis should become the standard of care for all individuals undergoing transplantation. Following the guidelines of this document will lead to a significant reduction in the number of cases of active TB after transplantation.

\section{SUPPORT STATEMENT}

D. Bumbacea was supported by a grant from CNCSIS- UEFISCSU (grant PNII-IDEI 1447/2008).

\section{STATEMENT OF INTEREST}

Statements of interest for D. Bumbacea, D. Goletti, M.G. Ison, P. Muñoz and N. Theodoropoulos can be found at www.erj.ersjournals.com/ site/misc/statements.xhtml

\section{ACKNOWLEDGEMENTS}

Author names and affiliations are as follows (sections to which the author was the lead contributor are given in parentheses): D. Bumbacea (Introduction): Dept of Pneumology, Elias Emergency University Hospital and Carol Davila University of Medicine and Pharmacy, Bucharest, Romania. S.M. Arend (Natural history of tuberculosis infection in transplant candidates and recipients): Dept of Infectious Diseases, Leiden University Medical Center, Leiden, the Netherlands. F. Eyuboglu: Pulmonary Diseases Dept, Baskent University School of Medicine, Ankara, Turkey. J.A. Fishman (Special considerations in stem cell transplant recipients): Transplantation Infectious Disease and Compromised Host Program, Massachusetts General Hospital, Harvard Medical School, Boston, MA, USA. D. Goletti (Future research directions): National Institute for Infectious Diseases L. Spallanzani, Rome, Italy. M.G. Ison (Risk of tuberculosis in solid organ transplant recipients): North Western University Feinberg School of Medicine, Division of Organ Transplantation, Chicago, IL, USA. C.E. Jones (Special considerations in children): Imperial College, London, UK. B. Kampmann: Imperial College, London, UK. C.N. Kotton (Prevention of tuberculosis in transplant recipients): Transplantation Infectious Disease and Compromised Host Program, Massachusetts General Hospital, Harvard Medical School, Boston, MA, USA. C. Lange (Consensus Recommendations): Division of Clinical Infectious Diseases, Medical Clinic, Research Center Borstel, Borstel, Germany. P. Ljungman: Karolinska University Hospital and Karolinska Institutet, Stockholm, Sweden. H. Milburn (Published national guidelines): Dept of Respiratory Medicine, Guy's and St Thomas Hospital, London, UK. M.I. Morris: Division of Infectious Diseases, University of Miami Miller School of Medicine, Miami, FL, USA. E. Muller: Groote Schuur Hospital, Cape Town, South Africa. P. Muñoz (Treatment of active tuberculosis in transplant recipients): Hospital General Universitario Gregorio Marañón, Dept of Medicine, Complutense University of Madrid, Madrid, Spain. A. Nellore: Transplantation Infectious Disease and Compromised Host Program, Massachusetts General Hospital, Harvard Medical School, Boston, MA, USA. H.L. Rieder: International Union Against Tuberculosis and Lung Disease, Paris, France, and Institute of Social and Preventive Medicine, University of Zurich, Zurich, Switzerland. U. Sester: Dept of Internal Medicine IV, Saarland University, Homburg, Germany. N. Theodoropoulos: Northwestern University Feinberg School of Medicine, Division of Organ Transplantation, Chicago, IL, USA. D. Wagner: Center of Chronic Immunodeficiency and Dept of Infectious Diseases, University of Freiburg, Freiburg, Germany. M. Sester (Diagnosis of latent infection with $M$. tuberculosis in transplant candidates, donors and recipients; Conclusions): Dept of Transplant and Infection Immunology, Saarland University, Homburg, Germany.

\section{REFERENCES}

1 Flynn JL, Chan J. Immunology of tuberculosis. Annu Rev Immunol 2001; 19: 93-129.

2 Kaufmann SH, Cole ST, Mizrahi V, et al. Mycobacterium tuberculosis and the host response. J Exp Med 2005; 201: 1693-1697.

3 Singh N, Paterson DL. Mycobacterium tuberculosis infection in solid-organ transplant recipients: impact and implications for management. Clin Infect Dis 1998; 27: 1266-1277. 
4 Subramanian A, Dorman S. Mycobacterium tuberculosis in solid organ transplant recipients. Am J Transplant 2009; 9: Suppl. 4, S57-S62.

5 Roy V, Weisdorf D. Mycobacterial infections following bone marrow transplantation: a 20 year retrospective review. Bone Marrow Transplant 1997; 19: 467-470.

6 Aguado JM, Herrero JA, Gavalda J, et al. Clinical presentation and outcome of tuberculosis in kidney, liver, and heart transplant recipients in Spain. Spanish Transplantation Infection Study Group, GESITRA. Transplantation 1997; 63: 1278-1286.

7 Russo RL, Dulley FL, Suganuma L, et al. Tuberculosis in hematopoietic stem cell transplant patients: case report and review of the literature. Int J Infect Dis 2010; 14: Suppl. 3, e187-e191.

8 Kaufmann SH. Future vaccination strategies against tuberculosis: thinking outside the box. Immunity 2010; 33: 567-577.

9 Sester U, Fousse M, Dirks J, et al. Whole-blood flow-cytometric analysis of antigen-specific CD4 T-cell cytokine profiles distinguishes active tuberculosis from non-active states. PLoS One 2011; 6: e17813.

10 Foulds KE, Wu CY, Seder RA. Th1 memory: implications for vaccine development. Immunol Rev 2006; 211: 58-66.

11 Sutherland JS, Adetifa IM, Hill PC, et al. Pattern and diversity of cytokine production differentiates between Mycobacterium tuberculosis infection and disease. Eur J Immunol 2009; 39: 723-729.

12 Caccamo N, Guggino G, Joosten SA, et al. Multifunctional CD4(+) T cells correlate with active Mycobacterium tuberculosis infection. Eur J Immunol 2010; 40: 2211-2220.

13 Harari A, Rozot V, Enders FB, et al. Dominant TNF- $\alpha+$ Mycobacterium tuberculosis-specific CD4+ T cell responses discriminate between latent infection and active disease. Nat Med 2011; 17: 372-376.

14 Mack U, Migliori GB, Sester M, et al. LTBI: latent tuberculosis infection or lasting immune responses to $M$. tuberculosis? A TBNET consensus statement. Eur Respir J 2009; 33: 956-973.

15 Leyten EM, Lin MY, Franken KL, et al. Human T-cell responses to 25 novel antigens encoded by genes of the dormancy regulon of Mycobacterium tuberculosis. Microbes Infect 2006; 8: 2052-2060.

16 Voskuil MI, Visconti KC, Schoolnik GK. Mycobacterium tuberculosis gene expression during adaptation to stationary phase and low-oxygen dormancy. Tuberculosis (Edinb) 2004; 84: 218-227.

17 Comstock GW, Livesay VT, Woolpert SF. The prognosis of a positive tuberculin reaction in childhood and adolescence. Am J Epidemiol 1974; 99: 131-138.

18 Vynnycky E, Fine PE. Lifetime risks, incubation period, and serial interval of tuberculosis. Am J Epidemiol 2000; 152: 247-263.

19 Lillebaek T, Dirksen A, Baess I, et al. Molecular evidence of endogenous reactivation of Mycobacterium tuberculosis after 33 years of latent infection. J Infect Dis 2002; 185: 401-404.

20 Arend SM, van Dissel JT. Evidence of endogenous reactivation of tuberculosis after a long period of latency. J Infect Dis 2002; 186: 876-877.

21 van de Vosse E, van Dissel JT, Ottenhoff TH. Genetic deficiencies of innate immune signalling in human infectious disease. Lancet Infect Dis 2009; 9: 688-698.

22 van der Eijk EA, van de Vosse E, Vandenbroucke JP, et al. Heredity versus environment in tuberculosis in twins: the 1950s United Kingdom Prophit Survey Simonds and Comstock revisited. Am J Respir Crit Care Med 2007; 176: 1281-1288.

23 Sutherland I, Švandová E, Radhakrishna S. The development of clinical tuberculosis following infection with tubercle bacilli. 1. A theoretical model for the development of clinical tuberculosis following infection, linking from data on the risk of tuberculous infection and the incidence of clinical tuberculosis in the Netherlands. Tubercle 1982; 63: 255-268.

24 Winthrop KL, Kubak BM, Pegues DA, et al. Transmission of Mycobacterium tuberculosis via lung transplantation. Am J Transplant 2004; 4: 1529-1533.
25 Centers for Disease Control and Prevention. Transplantationtransmitted tuberculosis - Oklahoma and Texas, 2007. MMWR Morb Mortal Wkly Rep 2008; 57: 333-336.

26 Edathodu J, Alrajhi A, Halim M, et al. Multi-recipient donortransmitted tuberculosis. Int J Tuberc Lung Dis 2010; 14: 1493-1495.

27 Smink F, van Hoek B, Ringers J, et al. Risk factors of acute hepatic failure during antituberculosis treatment: two cases and literature review. Neth J Med 2006; 64: 377-384.

28 Cardona PJ. New insights on the nature of latent tuberculosis infection and its treatment. Inflamm Allergy Drug Targets 2007; 6: 27-39.

29 Canetti G. Les réinfections tuberculeuses latentes du poumon. Etude anatomo-pathologique, bactériologique et pathogénique des lésions tuberculeuses abortives autres que de primoinfection, Paris: Vigot Frères, 1939.

30 Aguado JM, Torre-Cisneros J, Fortun J, et al. Tuberculosis in solid-organ transplant recipients: consensus statement of the group for the study of infection in transplant recipients (GESITRA) of the Spanish Society of Infectious Diseases and Clinical Microbiology. Clin Infect Dis 2009; 48: 1276-1284.

31 Currie AC, Knight SR, Morris PJ. Tuberculosis in renal transplant recipients: the evidence for prophylaxis. Transplantation 2010; 90: 695-704.

32 World Health Organization. Global Tuberculosis Control 2011, Geneva: WHO, 2011.

33 Torre-Cisneros J, Doblas A, Aguado JM, et al. Tuberculosis after solid-organ transplant: incidence, risk factors, and clinical characteristics in the RESITRA (Spanish Network of Infection in Transplantation) cohort. Clin Infect Dis 2009; 48: 1657-1665.

34 Borgdorff MW, Veen J, Kalisvaart NA, et al. Mortality among tuberculosis patients in the Netherlands in the period 1993-1995. Eur Respir J 1998; 11: 816-820.

35 Hall CM, Willcox PA, Swanepoel CR, et al. Mycobacterial infection in renal transplant recipients. Chest 1994; 106: 435-439.

36 Barry CE 3rd, Boshoff HI, Dartois V, et al. The spectrum of latent tuberculosis: rethinking the biology and intervention strategies. Nat Rev Microbiol 2009; 7: 845-855.

37 von Pirquet C. Die Allergieprobe zur Diagnose der Tuberkulose im Kindesalter. Wien Med Wochenschr 1907; 57: 1370-1374.

38 Sokal JE. Measurement of delayed skin-test responses. N Engl J Med 1975; 293: 501-502.

39 Targeted tuberculin testing and treatment of latent tuberculosis infection. American Thoracic Society. MMWR Recomm Rep 2000; 49: 1-51.

40 Cobelens FG, Egwaga SM, van Ginkel T, et al. Tuberculin skin testing in patients with HIV infection: limited benefit of reduced cutoff values. Clin Infect Dis 2006; 43: 634-639.

41 Lange C, Mori T. Advances in the diagnosis of tuberculosis. Respirology 2010; 15: 220-240.

42 Andersen P, Munk ME, Pollock JM, et al. Specific immune-based diagnosis of tuberculosis. Lancet 2000; 356: 1099-1104.

43 Mazurek GH, LoBue PA, Daley CL, et al. Comparison of a wholeblood interferon gamma assay with tuberculin skin testing for detecting latent Mycobacterium tuberculosis infection. JAMA 2001; 286: 1740-1747.

44 Lalvani A, Pathan AA, McShane $\mathrm{H}$, et al. Rapid detection of Mycobacterium tuberculosis infection by enumeration of antigenspecific T cells. Am J Respir Crit Care Med 2001; 163: 824-828.

45 Vincenti D, Carrara S, Butera O, et al. Response to region of difference 1 (RD1) epitopes in human immunodeficiency virus (HIV)-infected individuals enrolled with suspected active tuberculosis: a pilot study. Clin Exp Immunol 2007; 150: 91-98.

46 Diel R, Goletti D, Ferrara G, et al. Interferon-gamma release assays for the diagnosis of latent Mycobacterium tuberculosis infection: a systematic review and meta-analysis. Eur Respir J 2011; 37: 88-99. 
47 Sester M, Sotgiu G, Lange C, et al. Interferon- $\gamma$ release assays for the diagnosis of active tuberculosis: a systematic review and meta-analysis. Eur Respir J 2011; 37: 100-111.

48 Pai M, Zwerling A, Menzies D. Systematic review: T-cell-based assays for the diagnosis of latent tuberculosis infection: an update. Ann Intern Med 2008; 149: 177-184.

49 Mahairas GG, Sabo PJ, Hickey MJ, et al. Molecular analysis of genetic differences between Mycobacterium bovis BCG and virulent M. bovis. J Bacteriol 1996; 178: 1274-1282.

50 Gey van Pittius NC, Sampson SL, Lee H, Kim Y, et al. Evolution and expansion of the Mycobacterium tuberculosis PE and PPE multigene families and their association with the duplication of the ESAT-6 (esx) gene cluster regions. BMC Evol Biol 2006; 6: 95.

51 Lalvani A. Diagnosing tuberculosis infection in the 21st century: new tools to tackle an old enemy. Chest 2007; 131: 1898-1906.

52 Huebner RE, Schein MF, Bass JB Jr. The tuberculin skin test. Clin Infect Dis 1993; 17: 968-975.

53 Sester U, Junker H, Hodapp T, et al. Improved efficiency in detecting cellular immunity towards $M$. tuberculosis in patients receiving immunosuppressive drug therapy. Nephrol Dial Transplant 2006; 21: 3258-3268.

54 Munoz P, Rodriguez C, Bouza E. Mycobacterium tuberculosis infection in recipients of solid organ transplants. Clin Infect Dis 2005; 40: 581-587.

55 Halloran PF. Immunosuppressive drugs for kidney transplantation. N Engl J Med 2004; 351: 2715-2729.

56 Girndt M, Sester M, Sester U, et al. Defective expression of B7-2 (CD86) on monocytes of dialysis patients correlates to the uremia-associated immune defect. Kidney Int 2001; 59: 1382-1389.

57 Girndt M, Sester M, Sester U, et al. Molecular aspects of T- and Bcell function in uremia. Kidney Int Suppl 2001; 78: S206-S211.

58 Sester U, Wilkens H, van Bentum K, et al. Impaired detection of Mycobacterium tuberculosis immunity in patients using high levels of immunosuppressive drugs. Eur Respir J 2009; 34: 702-710.

59 Lange B, Vavra M, Kern WV, et al. Indeterminate results of a tuberculosis-specific interferon-gamma release assay in immunocompromised patients. Eur Respir J 2010; 35: 1179-1182.

60 Leung CC, Rieder HL, Lange C, et al. Treatment of latent infection with Mycobacterium tuberculosis: update 2010. Eur Respir J 2011; 37: 690-711.

61 Lange C, Rieder HL. Intention to test is intention to treat. Am J Respir Crit Care Med 2011; 183: 3-4.

62 Manuel O, Humar A, Preiksaitis J, et al. Comparison of Quantiferon-TB Gold with tuberculin skin test for detecting latent tuberculosis infection prior to liver transplantation. Am J Transplant 2007; 7: 2797-2801.

63 Lindemann M, Dioury Y, Beckebaum S, et al. Diagnosis of tuberculosis infection in patients awaiting liver transplantation. Hum Immunol 2009; 70: 24-28.

64 Sester M, Sester U, Clauer $\mathrm{P}$, et al. Tuberculin skin testing underestimates a high prevalence of latent tuberculosis infection in hemodialysis patients. Kidney Int 2004; 65: 1826-1834.

65 Passalent L, Khan K, Richardson R, et al. Detecting latent tuberculosis infection in hemodialysis patients: a head-to-head comparison of the T-SPOT.TB test, tuberculin skin test, and an expert physician panel. Clin J Am Soc Nephrol 2007; 2: 68-73.

66 Winthrop KL, Nyendak M, Calvet $H$, et al. Interferon-gamma release assays for diagnosing Mycobacterium tuberculosis infection in renal dialysis patients. Clin J Am Soc Nephrol 2008; 3: 1357-1363.

67 Lee SS, Chou KJ, Su IJ, et al. High prevalence of latent tuberculosis infection in patients in end-stage renal disease on hemodialysis: comparison of QuantiFERON-TB GOLD, ELISPOT, and tuberculin skin test. Infection 2009; 37: 96-102.

68 Triverio PA, Bridevaux PO, Roux-Lombard P, et al. Interferongamma release assays versus tuberculin skin testing for detection of latent tuberculosis in chronic haemodialysis patients. Nephrol Dial Transplant 2009; 24: 1952-1956.
69 Diel R, Loddenkemper R, Meywald-Walter K, et al. Predictive value of a whole blood IFN-gamma assay for the development of active tuberculosis disease after recent infection with Mycobacterium tuberculosis. Am J Respir Crit Care Med 2008; 177: 1164-1170.

70 Diel R, Loddenkemper R, Niemann S, et al. Negative and positive predictive value of a whole-blood interferon- $\gamma$ release assay for developing active tuberculosis: an update. Am J Respir Crit Care Med 2011; 183: 88-95.

71 Aichelburg MC, Rieger A, Breitenecker F, et al. Detection and prediction of active tuberculosis disease by a whole-blood interferon-gamma release assay in HIV-1-infected individuals. Clin Infect Dis 2009; 48: 954-962.

72 Girardi E, Goletti D. Do IFN-gamma-release assays predict the risk of TB? New evidence from a study on patients with silicosis. Expert Rev Anti Infect Ther 2010; 8: 1105-1108.

73 Rangaka MX, Wilkinson KA, Glynn JR, et al. Predictive value of interferon-gamma release assays for incident active tuberculosis: a systematic review and meta-analysis. Lancet Infect Dis 2012; 12: $45-55$.

74 Kim SH, Lee SO, Park JB, et al. A prospective longitudinal study evaluating the usefulness of a T-cell-based assay for latent tuberculosis infection in kidney transplant recipients. Am J Transplant 2011; 11: 1927-1935.

75 Lange B, Vavra M, Kern WV, et al. Development of tuberculosis in immunocompromised patients with positive tuberculosisspecific interferon-gamma release assay. Int J Tuberc Lung Dis 2012; 16: 492-495.

76 Holty JE, Gould MK, Meinke L, et al. Tuberculosis in liver transplant recipients: a systematic review and meta-analysis of individual patient data. Liver Transpl 2009; 15: 894-906.

77 Agoglia L, Balbi E, Halpern M, et al. Tuberculosis in liver transplant recipients: prophylaxis in an endemic area. Transplant Proc 2011; 43: 199-202.

78 John GT, Shankar V. Mycobacterial infections in organ transplant recipients. Semin Respir Infect 2002; 17: 274-283.

79 Lyu J, Lee SG, Hwang S, et al. Chest CT is more likely to show latent tuberculosis foci than simple chest radiography in liver transplantation candidates. Liver Transpl 2011; 17: 963-968.

80 Centers for Disease Control and Prevention. Update: Fatal and severe liver injuries associated with rifampin and pyrazinamide for latent tuberculosis infection., and revisions in American Thoracic Society/CDC recommendations - United States, 2001. MMWR Morb Mortal Wkly Rep 2001; 50: 733-735.

81 Centers for Disease Control and Prevention. Fatal and severe hepatitis associated with rifampin and pyrazinamide for the treatment of latent tuberculosis infection - New York and Georgia, 2000. MMWR Morb Mortal Wkly Rep 2001; 50: 289-291.

82 Cook PP, Maldonado RA, Yarnell CT, et al. Safety and completion rate of short-course therapy for treatment of latent tuberculosis infection. Clin Infect Dis 2006; 43: 271-275.

83 Stout JE. Safety of rifampin and pyrazinamide for the treatment of latent tuberculosis infection. Expert Opin Drug Saf 2004; 3: 187-198.

84 Targeted tuberculin testing and treatment of latent tuberculosis infection. Am J Respir Crit Care Med 2000; 161: S221-S247.

85 Centers for Disease Control and Prevention. Severe isoniazidassociated liver injuries among persons being treated for latent tuberculosis infection - United States, 2004-2008. MMWR Morb Mortal Wkly Rep 2010; 59: 224-229.

86 Page KR, Sifakis F, Montes de Oca R, et al. Improved adherence and less toxicity with rifampin $v s$ isoniazid for treatment of latent tuberculosis: a retrospective study. Arch Intern Med 2006; 166: 1863-1870.

87 Singh N, Wagener MM, Gayowski T. Safety and efficacy of isoniazid chemoprophylaxis administered during liver transplant candidacy for the prevention of posttransplant tuberculosis. Transplantation 2002; 74: 892-895. 
88 Jahng AW, Tran T, Bui L, et al. Safety of treatment of latent tuberculosis infection in compensated cirrhotic patients during transplant candidacy period. Transplantation 2007; 83: 1557-1562.

89 Taylor Z, Nolan CM, Blumberg HM. Controlling tuberculosis in the United States. Recommendations from the American Thoracic Society, CDC, and the Infectious Diseases Society of America. MMWR Recomm Rep 2005; 54: 1-81.

90 Chenhsu RY, Loong CC, Chou MH, et al. Renal allograft dysfunction associated with rifampin-tacrolimus interaction. Ann Pharmacother 2000; 34: 27-31.

91 Offermann G, Keller F, Molzahn M. Low cyclosporin A blood levels and acute graft rejection in a renal transplant recipient during rifampin treatment. Am J Nephrol 1985; 5: 385-387.

92 Cassidy MJ, Van Zyl-Smit R, Pascoe MD, et al. Effect of rifampicin on cyclosporin A blood levels in a renal transplant recipient. Nephron 1985; 41: 207-208.

93 Ngo BT, Pascoe M, Khan D. Drug interaction between rifampicin and sirolimus in transplant patients. Saudi J Kidney Dis Transpl 2011; 22: 112-115.

94 Vachharajani $\mathrm{T}$, Abreo K, Phadke A, et al. Diagnosis and treatment of tuberculosis in hemodialysis and renal transplant patients. Am J Nephrol 2000; 20: 273-277.

95 al-Sulaiman MH, Dhar JM, al-Khader AA. Successful use of rifampicin in the treatment of tuberculosis in renal transplant patients immunosuppressed with cyclosporine. Transplantation 1990; 50: 597-298.

96 Blumberg HM, Burman WJ, Chaisson RE, et al. American Thoracic Society/Centers for Disease Control and Prevention/ Infectious Diseases Society of America: treatment of tuberculosis. Am I Respir Crit Care Med 2003; 167: 603-662.

97 National Institute for Health and Clinical Excellence. Tuberculosis: clinical diagnosis and management of tuberculosis, and measures for its prevention and control, 2011 www.nice.org. uk/nicemedia/live/13422/53642/53642.pdf

98 Canet E, Dantal J, Blancho G, et al. Tuberculosis following kidney transplantation: clinical features and outcome. A French multicentre experience in the last 20 years. Nephrol Dial Transplant 2011; 26: 3773-3778.

99 Meyers BR, Papanicolaou GA, Sheiner P, et al. Tuberculosis in orthotopic liver transplant patients: increased toxicity of recommended agents; cure of disseminated infection with nonconventional regimens. Transplantation 2000; 69: 64-69.

100 Yew WW, Lange C, Leung CC. Treatment of tuberculosis: update 2010. Eur Respir J 2011; 37: 441-462.

101 Ziganshina LE, Squire SB. Fluoroquinolones for treating tuberculosis. Cochrane Database Syst Rev 2008; 1: CD004795.

102 Dorman SE, Johnson JL, Goldberg S, et al. Substitution of moxifloxacin for isoniazid during intensive phase treatment of pulmonary tuberculosis. Am J Respir Crit Care Med 2009; 180: 273-280.

103 Lou HX, Shullo MA, McKaveney TP. Limited tolerability of levofloxacin and pyrazinamide for multidrug-resistant tuberculosis prophylaxis in a solid organ transplant population. Pharmacotherapy 2002; 22: 701-704

104 Caminero JA, Sotgiu G, Zumla A, et al. Best drug treatment for multidrug-resistant and extensively drug-resistant tuberculosis. Lancet Infect Dis 2010; 10: 621-629.

105 Coca NS, Oliveira MS, Voieta I, et al. Antituberculosis druginduced hepatotoxicity: a comparison between patients with and without human immunodeficiency virus seropositivity. Rev Soc Bras Med Trop 2010; 43: 624-628.

106 Yew WW, Leung CC. Antituberculosis drugs and hepatotoxicity. Respirology 2006; 11: 699-707.

107 Place S, Knoop C, Remmelink M, et al. Paradoxical worsening of tuberculosis in a heart-lung transplant recipient. Transpl Infect Dis 2007; 9: 219-224.
108 Calligaro G, Meintjes G, Mendelson M. Pulmonary manifestations of the immune reconstitution inflammatory syndrome. Curr Opin Pulm Med 2011; 17: 180-188.

109 Sun HY, Singh N. Immune reconstitution inflammatory syndrome in non-HIV immunocompromised patients. Curr Opin Infect Dis 2009; 22: 394-402.

110 Karras A, Thervet E, Legendre C. Hemophagocytic syndrome in renal transplant recipients: report of 17 cases and review of literature. Transplantation 2004; 77: 238-243.

111 Di Perri G, Luzzati R, Forni A, et al. Fatal primary multidrugresistant tuberculosis in a heart transplant recipient. Transpl Int 1998; 11: 305-307.

112 Lee J, Yew WW, Wong CF, et al. Multidrug-resistant tuberculosis in a lung transplant recipient. J Heart Lung Transplant 2003; 22 1168-1173.

113 Shitrit D, Bendayan D, Saute M, et al. Multidrug resistant tuberculosis following lung transplantation: treatment with pulmonary resection. Thorax 2004; 59: 79-80.

114 Altclas J, Lescano A, Salgueira C, et al. Multidrug-resistant tuberculosis in bone marrow transplant recipient. Transpl Infect Dis 2005; 7: 45-46.

115 World Health Organization. Guidelines for the programmatic management of drug-resistant tuberculosis. 2011 update, Geneva: WHO, 2011.

116 Yew WW. Management of multidrug-resistant tuberculosis and extensively drug-resistant tuberculosis: current status and future prospects. Kekkaku 2011; 86: 9-16.

117 Lee J, Lee MH, Kim WS, et al. Tuberculosis in hematopoietic stem cell transplant recipients in Korea. Int J Hematol 2004; 79: 185-188.

118 Budak-Alpdogan T, Tangun Y, Kalayoglu-Besisik S, et al. The frequency of tuberculosis in adult allogeneic stem cell transplant recipients in Turkey. Biol Blood Marrow Transplant 2000; 6: 370-374

119 Ip MSM, Yuen KY, Woo PCY, et al. Risk factors for pulmonary tuberculosis in bone marrow transplant recipients. Am J Respir Crit Care Med 1998; 158: 1173-1177.

120 Khan B, Ahmed P, Ullah K, et al. Frequency of tuberculosis in haematological malignancies and stem cell transplant recipients J Coll Physicians Surg Pak 2005; 15: 30-33.

121 Navari RM, Sullivan KM, Springmeyer SC, et al. Mycobacterial infections in marrow transplant patients. Transplantation 1983, 36: 509-513.

122 Erdstein AA, Daas P, Bradstock KF, et al. Tuberculosis in allogeneic stem cell transplant recipients: still a problem in the 21st century. Transpl Infect Dis 2004; 6: 142-146.

123 Ambrossi G, Jkubowski, A, Feinstein MG, et al. Active tuberculosis limited to foreign-born patients after allogeneic hematopoietic stem cell transplant. Bone Marrow Transplant 2005; 36: 3.

124 de la Camara R, Martino R, Granados E, et al. Tuberculosis after hematopoietic stem cell transplantation: incidence, clinical characteristics and outcome. Spanish Group on Infectious Complications in Hematopoietic Transplantation. Bone Marrow Transplant 2000; 26: 291-298.

125 Cordonnier C, Martino R, Trabasso P, et al. Mycobacterial infection: a difficult and late diagnosis in stem cell transplant recipients. Clin Infect Dis 2004; 38: 1229-1236.

$126 \mathrm{Ku} \mathrm{SC}$, Tang JL, Hsueh PR, et al. Pulmonary tuberculosis in allogeneic hematopoietic stem cell transplantation. Bone Marrow Transplant 2001; 27: 1293-1297.

127 Jung JI, Lee DG, Kim YJ, et al. Pulmonary tuberculosis after hematopoietic stem cell transplantation: radiologic findings. J Thorac Imaging 2009; 24: 10-16.

128 Saadeh CE, Srkalovic G. Mycobacterium avium complex infection after alemtuzumab therapy for chronic lymphocytic leukemia Pharmacotherapy 2008; 28: 281-284

129 Martino R, Martinez C, Brunet $S$, et al. Tuberculosis in bone marrow transplant recipients: report of two cases and review of the literature. Bone Marrow Transplant 1996; 18: 809-812. 
130 Rouleau M, Senik A, Leroy E, et al. Long-term persistence of transferred PPD-reactive $\mathrm{T}$ cells after allogeneic bone marrow transplantation. Transplantation 1993; 55: 72-76.

131 Halaburda K, Marianska B, Warzocha K, et al. Clinical evaluation of busulfan, cladribine and alemtuzumab as reduced intensity conditioning for stem cell transplantation. Ann Transplant 2009; 14: 7-12.

132 Kim SJ, Lee JW, Jung CW, et al. Weekly rituximab followed by monthly rituximab treatment for steroid-refractory chronic graft versus host disease: results from a prospective, multicenter, phase II study. Haematologica 2010; 95: 1935-1942.

133 Tam LS, Leung CC, Ying SK, et al. Risk of tuberculosis in patients with rheumatoid arthritis in Hong Kong: the role of TNF blockers in an area of high tuberculosis burden. Clin Exp Rheumatol 2010; 28: 679-685.

134 Keane J, Gershon S, Wise RP, et al. Tuberculosis associated with infliximab, a tumor necrosis factor $\alpha$-neutralizing agent. $N$ Engl J Med 2001; 345: 1098-1104.

135 National Tuberculosis Controllers Association. Centers for Disease Control and Prevention. Guidelines for the investigation of contacts of persons with infectious tuberculosis. Recommendations from the National Tuberculosis Controllers Association and CDC. MMWR Recomm Rep 2005; 54: 1-47.

136 Kobashi Y, Mouri K, Obase Y, et al. Clinical evaluation of QuantiFERON TB-2G test for immunocompromised patients. Eur Respir J 2007; 30: 945-950.

137 Canadian Tuberculosis Committee. Interferon gamma release assays for latent tuberculosis infection. An Advisory Committee Statement (ACS). Can Commun Dis Rep 2007; 33: 1-18.

138 Tomblyn M, Chiller T, Einsele H, et al. Guidelines for preventing infectious complications among hematopoietic cell transplantation recipients: a global perspective. Biol Blood Marrow Transplant 2009; 15: 1143-1238.

139 Ljungman P, Cordonnier C, Einsele H, et al. Vaccination of hematopoietic cell transplant recipients. Bone Marrow Transplant 2009; 44: 521-526.

140 Spearman CW, McCulloch M, Millar AJ, et al. Liver transplantation at Red Cross War Memorial Children's Hospital. S Afr Med J 2006; 96: 960-963.

141 Verma A, Dhawan A, Wade JJ, et al. Mycobacterium tuberculosis infection in pediatric liver transplant recipients. Pediatr Infect Dis J 2000; 19: 625-630.

142 McCulloch MI, Gajjar P, Spearman CW, et al. Overview of a paediatric renal transplant programme. S Afr Med J 2006; 96: 955-959.

143 Rizvi SA, Naqvi SA, Hussain Z, et al. Living-related pediatric renal transplants: a single-center experience from a developing country. Pediatr Transplant 2002; 6: 101-110.

144 George B, Mathews V, Viswabandya A, et al. Infections in children undergoing allogeneic bone marrow transplantation in India. Pediatr Transplant 2006; 10: 48-54.

145 Cruz AT, Starke JR. Clinical manifestations of tuberculosis in children. Paediatr Respir Rev 2007; 8: 107-117.

146 Marais BJ, Gie RP, Schaaf HS, et al. The clinical epidemiology of childhood pulmonary tuberculosis: a critical review of literature from the pre-chemotherapy era. Int J Tuberc Lung Dis 2004; 8: 278-285.

147 Newton SM, Brent AJ, Anderson S, et al. Paediatric tuberculosis. Lancet Infect Dis 2008; 8: 498-510.

148 McDiarmid SV, Blumberg DA, Remotti H, et al. Mycobacterial infections after pediatric liver transplantation: a report of three cases and review of the literature. J Pediatr Gastroenterol Nutr 1995; 20: 425-431.

149 Diel R, Forssbohm M, Loytved G, et al. Empfehlungen fur die Umgebungsuntersuchungen bei Tuberkulose [Recommendations for background studies in tuberculosis]. Pneumologie 2007; 61: $440-455$.
150 Diel R, Forssbohm M, Loytved G, et al. Empfehlungen für die Umgebungsuntersuchungen bei Tuberkulose. Deutsches Zentralkomitee zur Bekampfung der Tuberkulose [Recommendations for environmental contact tracing in tuberculosis. German Central Committee against Tuberculosis]. Gesundheitswesen 2007; 69: 488-503.

151 Nicol MP, Zar HJ. New specimens and laboratory diagnostics for childhood pulmonary TB: progress and prospects. Paediatr Respir Rev 2011; 12: 16-21.

152 Knoll G, Cockfield S, Blydt-Hansen T, et al. Canadian Society of Transplantation consensus guidelines on eligibility for kidney transplantation. CMAJ 2005; 173: 1181-1184.

153 EBPG Expert Group on Renal Transplantation. European best practice guidelines for renal transplantation. Section IV: Longterm management of the transplant recipient. IV.7.2. Late infections. Tuberculosis. Nephrol Dial Transplant 2002; 17: Suppl. 4, 39-43.

154 Milburn H, Ashman N, Davies P, et al. Guidelines for the prevention and management of Mycobacterium tuberculosis infection and disease in adult patients with chronic kidney disease. Thorax 2010; 65: 557-570.

155 KDIGO clinical practice guideline for the care of kidney transplant recipients. Am J Transplant 2009; 9: Suppl. 3, S1-155.

$156 \mathrm{Al}$ Jahdali $\mathrm{HH}$, Baharoon $\mathrm{S}$, Abba AA, et al. Saudi guidelines for testing and treatment of latent tuberculosis infection. Ann Saudi Med 2010; 30: 38-49.

157 Haddad H, Isaac D, Legare JF, et al. Canadian Cardiovascular Society Consensus Conference update on cardiac transplantation 2008: Executive Summary. Can J Cardiol 2009; 25: 197-205.

158 Infection. In: Kälble TAlcaraz ABudde $\mathrm{K}$, et al. Guidelines on Renal Transplantation, Arnhem: European Association of Urology, 2009; p. 32.

159 Smieja MJ, Marchetti CA, Cook DJ, et al. Isoniazid for preventing tuberculosis in non-HIV infected persons. Cochrane Database Syst Rev 2000; 2: CD001363.

160 Boehme CC, Nicol MP, Nabeta P, et al. Feasibility, diagnostic accuracy, and effectiveness of decentralised use of the Xpert MTB/RIF test for diagnosis of tuberculosis and multidrug resistance: a multicentre implementation study. Lancet 2011; 377: 1495-1505.

161 Boehme CC, Nabeta P, Hillemann D, et al. Rapid molecular detection of tuberculosis and rifampin resistance. $N$ Engl J Med 2010; 363: 1005-1015

162 Theron G, Peter J, van Zyl-Smit R, et al. Evaluation of the Xpert MTB/RIF assay for the diagnosis of pulmonary tuberculosis in a high HIV prevalence setting. Am J Respir Crit Care Med 2011; 184: 132-140.

163 Ling DI, Pai M, Davids V, et al. Are interferon- $\gamma$ release assays useful for active tuberculosis in a high-burden setting? Eur Respir J 2011; 38: 649-656.

164 Black GF, Thiel BA, Ota MO, et al. Immunogenicity of novel DosR regulon-encoded candidate antigens of Mycobacterium tuberculosis in three high-burden populations in Africa. Clin Vaccine Immunol 2009; 16: 1203-1212.

165 Goletti D, Butera O, Vanini V, et al. Response to Rv2628 latency antigen associates with cured tuberculosis and remote infection Eur Respir J 2010; 36: 135-142.

166 Ruhwald M, Bjerregaard-Andersen M, Rabna P, et al. CXCL10/ IP-10 release is induced by incubation of whole blood from tuberculosis patients with ESAT-6, CFP10 and TB7.7. Microbes Infect 2007; 9: 806-812.

167 Ruhwald M, Bodmer T, Maier C, et al. Evaluating the potential of IP-10 and MCP-2 as biomarkers for the diagnosis of tuberculosis. Eur Respir J 2008; 32: 1607-1615.

168 Goletti D, Raja A, Syed Ahamed Kabeer B, et al. Is IP-10 an accurate marker for detecting $M$. tuberculosis-specific response in HIV-infected persons? PLoS One 2010; 5: e12577. 
169 Delogu G, Chiacchio T, Vanini V, et al. Methylated HBHA produced in $M$. smegmatis discriminates between active and nonactive tuberculosis disease among RD1-responders. PLoS One 2011; 6: e18315.

170 Millington KA, Fortune SM, Low J, et al. Rv3615c is a highly immunodominant RD1 (region of difference 1)-dependent secreted antigen specific for Mycobacterium tuberculosis infection. Proc Natl Acad Sci USA 2011; 108: 5730-5735.

171 Goletti D, Raja A, Ahamed Kabeer BS, et al. IFN-gamma, but not IP-10, MCP-2 or IL-2 response to RD1 selected peptides associates to active tuberculosis. J Infect 2010; 61: 133-143.

172 Kabeer BS, Raja A, Raman B, et al. IP-10 response to RD1 antigens might be a useful biomarker for monitoring tuberculosis therapy. BMC Infect Dis 2011; 11: 135.

173 Leyten EM, Arend SM, Prins C, et al. Discrepancy between Mycobacterium tuberculosis-specific gamma interferon release assays using short and prolonged in vitro incubation. Clin Vaccine Immunol 2007; 14: 880-885.

174 Butera O, Chiacchio T, Carrara S, et al. New tools for detecting latent tuberculosis infection: evaluation of RD1-specific longterm response. BMC Infect Dis 2009; 9: 182.

175 Goletti D, Butera O, Bizzoni F, et al. Region of difference 1 antigen-specific CD4+ memory T-cells correlate with a favorable outcome of tuberculosis. J Infect Dis 2006; 194: 984-992.

176 Chiacchio T, Petruccioli E, Vanini V, et al. Higher frequency of Tcell response to M. tuberculosis latency antigen Rv2628 at the site of active tuberculosis disease than in peripheral blood. PLoS One 2011; 6: e27539.

177 Jafari C, Thijsen S, Sotgiu G, et al. Bronchoalveolar lavage enzyme-linked immunospot for a rapid diagnosis of tuberculosis: a Tuberculosis Network European Trials group study. Am J Respir Crit Care Med 2009; 180: 666-673.

178 Losi M, Bossink A, Codecasa L, et al. Use of a T-cell interferon- $\gamma$ release assay for the diagnosis of tuberculous pleurisy. Eur Respir J 2007; 30: 1173-1179.

179 Thomas MM, Hinks TS, Raghuraman S, et al. Rapid diagnosis of Mycobacterium tuberculosis meningitis by enumeration of cerebrospinal fluid antigen-specific T-cells. Int J Tuberc Lung Dis 2008; 12: 651-657.

180 Berry MP, Graham CM, McNab FW, et al. An interferoninducible neutrophil-driven blood transcriptional signature in human tuberculosis. Nature 2010; 466: 973-977.

181 Ginsberg AM. Tuberculosis drug development: progress, challenges, and the road ahead. Tuberculosis (Edinb) 2010; 90: 162-167.

182 Tomioka H, Tatano Y, Sano C, et al. Development of new antituberculous drugs based on bacterial virulence factors interfering with host cytokine networks. J Infect Chemother 2011; 17: 302-317.

183 Wallis RS. Reconsidering adjuvant immunotherapy for tuberculosis. Clin Infect Dis 2005; 41: 201-208.

184 Sterling TR, Villarino ME, Borisov AS, et al. Three months of rifapentine and isoniazid for latent tuberculosis infection. $N$ Engl J Med 2011; 365: 2155-2166. 